\title{
Impact of Sickle Cell Trait Hemoglobin on the Intraerythrocytic Transcriptional Program of Plasmodium falciparum
}

\author{
Joseph W. Saelens, ${ }^{a}$ Jens E. V. Petersen, ${ }^{a}$ Elizabeth Freedman, ${ }^{a}$ Robert C. Moseley, ${ }^{b}$ Drissa Konaté, ${ }^{c}$ Seidina A. S. Diakité, ${ }^{c}$ \\ Karim Traoré, ${ }^{c}$ Natalie Vance, ${ }^{d}$ Rick M. Fairhurst, ${ }^{e}$ Mahamadou Diakité, ${ }^{c}$ Steven B. Haase, ${ }^{\text {a,b }}$ D Steve M. Taylor ${ }^{a, d}$ \\ aDivision of Infectious Diseases, Duke University School of Medicine, Durham, North Carolina, USA \\ bDepartment of Biology, Duke University, Durham, North Carolina, USA \\ cMalaria Research and Training Center, Mali International Center for Excellence in Research, University of Sciences, Techniques and Technologies of Bamako, Bamako, \\ Mali \\ ¿Duke Global Health Institute, Duke University, Durham, North Carolina, USA \\ eLaboratory of Malaria and Vector Research, National Institute of Allergy and Infectious Diseases, National Institutes of Health, Bethesda, Maryland, USA
}

ABSTRACT Sickle-trait hemoglobin (HbAS) confers nearly complete protection from severe, life-threatening falciparum malaria in African children. Despite this clear protection, the molecular mechanisms by which HbAS confers these protective phenotypes remain incompletely understood. As a forward genetic screen for aberrant parasite transcriptional responses associated with parasite neutralization in HbAS red blood cells (RBCs), we performed comparative transcriptomic analyses of Plasmodium falciparum in normal ( $\mathrm{HbAA})$ and $\mathrm{HbAS}$ erythrocytes during both in vitro cultivation of reference parasite strains and naturally occurring $P$. falciparum infections in Malian children with $\mathrm{HbAA}$ or HbAS. During in vitro cultivation, parasites matured normally in HbAS RBCs, and the temporal expression was largely unperturbed of the highly ordered transcriptional program that underlies the parasite's maturation throughout the intraerythrocytic development cycle (IDC). However, differential expression analysis identified hundreds of transcripts aberrantly expressed in HbAS, largely occurring late in the IDC. Surprisingly, transcripts encoding members of the Maurer's clefts were overexpressed in HbAS despite impaired parasite protein export in these RBCs, while parasites in HbAS RBCs underexpressed transcripts associated with the endoplasmic reticulum and those encoding serine repeat antigen proteases that promote parasite egress. Analyses of $P$. falciparum transcriptomes from 32 children with uncomplicated malaria identified stage-specific differential expression: among infections composed of ring-stage parasites, only cyclophilin 19B was underexpressed in children with $\mathrm{HbAS}$, while trophozoite-stage infections identified a range of differentially expressed transcripts, including downregulation in HbAS of several transcripts associated with severe malaria in collateral studies. Collectively, our comparative transcriptomic screen in vitro and in vivo indicates that $P$. falciparum adapts to HbAS by altering its protein chaperone and folding machinery, oxidative stress response, and protein export machinery. Because HbAS consistently protects from severe $P$. falciparum, modulation of these responses may offer avenues by which to neutralize $P$. falciparum parasites.

IMPORTANCE Sickle-trait hemoglobin (HbAS) confers nearly complete protection from severe, life-threatening malaria, yet the molecular mechanisms that underlie HbAS protection from severe malaria remain incompletely understood. Here, we used transcriptome sequencing (RNA-seq) to measure the impact of HbAS on the blood-stage transcriptome of Plasmodium falciparum in in vitro time series experiments and in vivo samples from natural infections. Our in vitro time series data reveal that, during its blood stage, $P$. falciparum's gene expression in HbAS is impacted primarily through alterations in the abundance of gene products as opposed to variations in
Editor Ira J. Blader, University at Buffalo Copyright $\odot 2021$ Saelens et al. This is an open-access article distributed under the terms of the Creative Commons Attribution 4.0 International license. Address correspondence to Steve M. Taylor steve.taylor@duke.edu.

Received 21 September 2021 Accepted 29 September 202 Published 20 October 2021 
the timing of gene expression. Collectively, our in vitro and in vivo data indicate that $P$. falciparum adapts to $\mathrm{HbAS}$ by altering its protein chaperone and folding machinery, oxidative stress response, and protein export machinery. Due to the persistent association of $\mathrm{HbAS}$ and protection from severe disease, these processes that are modified in HbAS may offer strategies to neutralize $P$. falciparum.

KEYWORDS comparative studies, malaria, stress adaptation, transcriptional regulation

$\mathrm{n}$ regions across Africa where malaria is endemic, red blood cell (RBC) variants are highly prevalent and can confer significant protection against severe malaria (1). Heterozygosity for sickle hemoglobin $(\mathrm{Hb})$ provides one striking example of the protection afforded by an RBC variant: sickle-cell trait (HbAS) reduces the risk of severe, lifethreatening malaria by over $90 \%$ (2). The functional underpinnings of this extensive protection afforded by HbAS against severe disease hold potential for uncovering mechanisms of parasite pathogenesis that can be exploited by future interventions.

Candidate protective mechanisms reported thus far are diverse and provide evidence that HbAS disrupts mediators of pathogenesis and the parasite life cycle in infected RBCs (iRBCs). Parasite maturation may be inhibited by either low oxygen tension (3-6) or host microRNAs (miRNAs) that are enriched in HbSS and HbAS RBCs (7), though others have reported equivalent growth in HbAA and HbAS iRBCs under the same conditions $(8,9)$. Multiple lines of evidence support a mechanism in which cytoadherence, a central property of Plasmodium falciparum's pathogenesis, is reduced in $\mathrm{HbAS}$ iRBCs. HbAS reduces both the display on the $\mathrm{iRBC}$ surface of $P$. falciparum erythrocyte membrane protein 1 (PfEMP-1) and knob density (10). This is supported by the observation that HbAS impairs export of proteins, including PfEMP-1, to the iRBC surface via Maurer's clefts, which sort and traffic parasite proteins (11). The attenuated export of surface proteins and concomitant reduction in cytoadherence would compromise the capacity of $\mathrm{HbAS}$ iRBCs to sequester in the microvasculature endothelium, thereby diminishing endothelial activation-as has been observed in vitro (12) - as well as subsequent events in the microvasculature that can lead to severe disease in vivo (13). These downstream phenotypes of HbAS iRBCs are the result of incompletely understood upstream parasite responses to the altered RBC environment.

The natural protection against severe falciparum malaria conferred by HbAS provides an opportunity to identify parasite mechanisms that are neutralized and therefore critical to pathogenesis. In this study, we used transcriptome sequencing (RNAseq) to perform an unbiased comparative transcriptomic survey of the $P$. falciparum transcriptional changes that occur in HbAS iRBCs. Owing to the reduced cytoadherence observed in HbAS iRBCs, we hypothesized that gene products functioning in cytoadherence would be downregulated in HbAS. Therefore, we investigated parasite transcription over the intraerythrocytic development cycle (IDC) in vitro in tightly synchronized parasite populations in $\mathrm{HbAA}$ and $\mathrm{HbAS}$ iRBCs as well as in freshly collected parasites from Malian children with uncomplicated malaria with HbAA or HbAS.

\section{RESULTS}

Parasite maturation and transcriptome characteristics. We interrogated two reference Plasmodium falciparum strains that originated where the sickle allele of $\beta$-globin is common (14): 3D7 from West Africa (15) and FUP from Uganda (16). Based on morphology assessed by microscopy, parasites matured similarly in $\mathrm{HbAA}$ and $\mathrm{HbAS}$ iRBCs for both 3D7 and FUP (Fig. 1A). Parasites in HbAA and HbAS iRBCs transitioned from rings to trophozoites at $27 \mathrm{~h}$ postinvasion (hpi) (3D7) and $21 \mathrm{hpi}$ (FUP).

Principal-component analysis (PCA) based on transcript counts clustered samples by parasite line and developmental stage (Fig. 1B). Notably, the principal components that explain the vast majority of the transcriptional variance between samples did not separate parasite transcriptomes in HbAS iRBCs from those in HbAA iRBCs. Similarly, visualization of normalized expression of 5,499 parasite transcripts suggests that the progression of $P$. falciparum's IDC transcriptional program remains largely intact in 
A

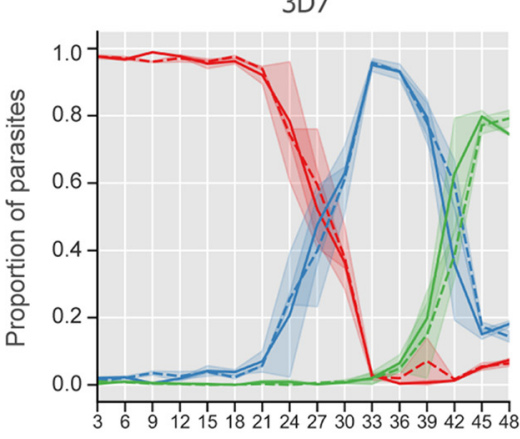

Hours post-invasion
FUP

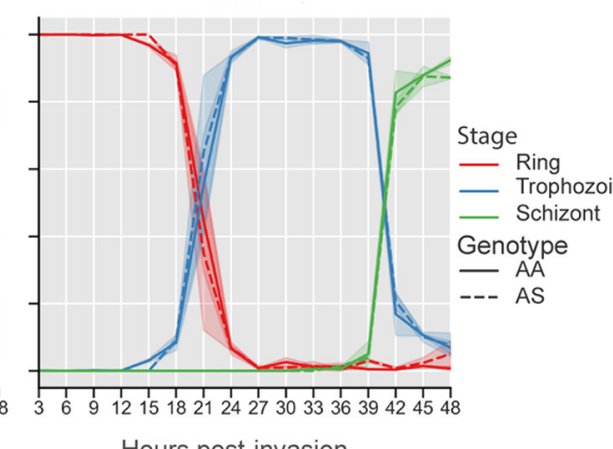

Hours post-invasion

B

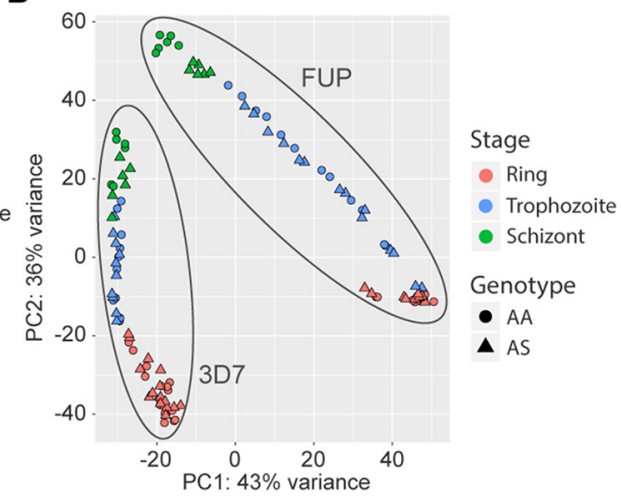

FUP

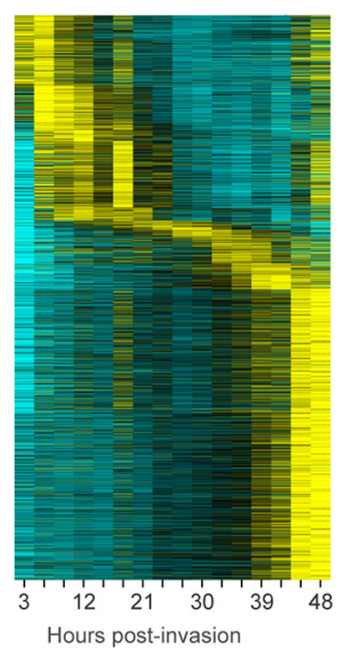

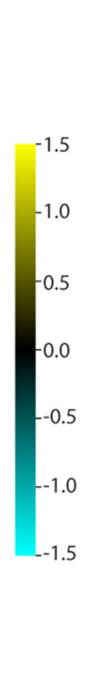

$\mathrm{HbAA}$

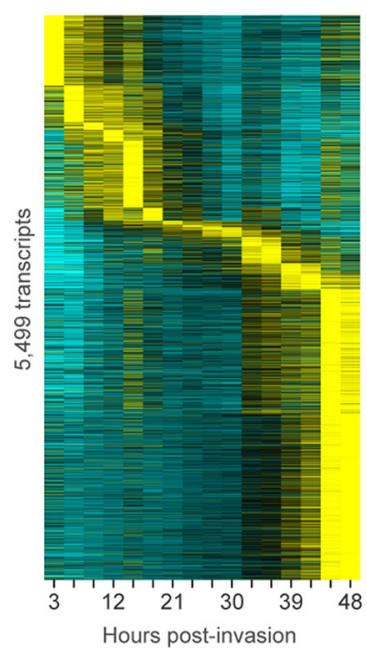

3D7
HbAA

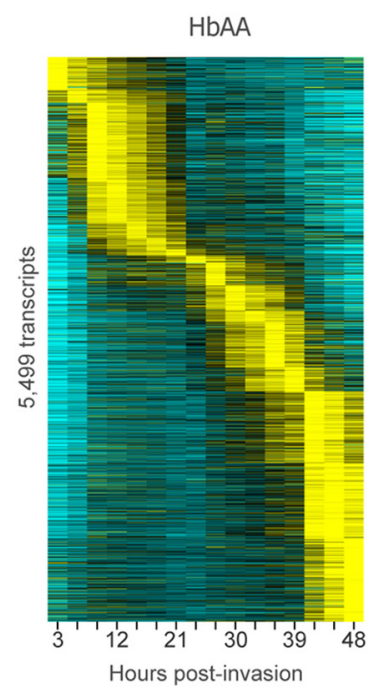

HbAS

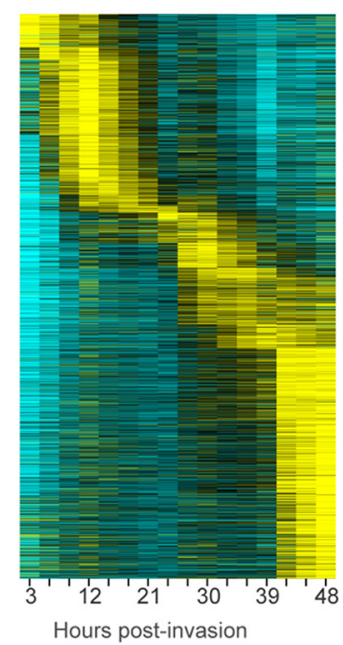

FIG 1 P. falciparum maturation and the overall transcriptional program are conserved in HbAS iRBCs. (A) The relative abundance of ring, trophozoite, and schizont stage parasites from light microscopy readings in HbAA (solid line) and HbAS (dotted line) iRBCs. 3D7 and FUP parasites mature at similar rates in $\mathrm{HbAA}$ and HbAS iRBCs. Counts are based on 500 parasites from each sample per time point and were assessed by a reader masked to time point and RBC type. (B) Principal-component analysis of transcript expression following variance-stabilizing transformation based on the 500 transcripts with the highest variance across all samples. Samples cluster according to parasite strain and developmental stage. (C) Expression of 5,499 transcripts passing thresholds in the ASM276v2 transcriptome in 3D7 and FUP isolates. In both 3D7 and FUP, the ordering of expression is largely conserved between HbAA and HbAS samples. Transcripts are ordered vertically by the peak expression time in HbAA RBCs for each parasite strain. The columns along the $x$ axis depict the normalized expression values of each transcript per time point. Expression values were averaged across replicates and are normalized around the mean for each transcript over the time series and depicted as a $z$ score of standard deviations from the mean. Values range between -1.5 (cyan) and 1.5 (yellow). Scripts for RNA-seq read preparation and quantification are available on our GitHub page.

HbAS iRBCs (Fig. 1C). These data indicate both that the parasite populations in each sample were highly synchronous and that HbAS does not broadly disrupt $P$. falciparum's transcriptional program during the IDC.

Transcriptional synchrony of parasites in HbAS RBCs. $P$. falciparum maturation in HbAS iRBCs was described previously to be delayed compared to that in HbAA iRBCs (6). To assess parasite maturation at a finer scale, we computed, for the subset of transcripts that had a single transcription peak, the temporal shift of each transcript's expression peak in HbAS iRBCs compared to HbAA (Fig. 2A). The distribution of peak shifts between $\mathrm{HbAA}$ and $\mathrm{HbAS}$ over the entire time course was centered around zero for both 3D7 and FUP (Fig. 2B), with slight but nonsignificant mean peak shift delays in HbAS RBCs for both 3D7 ( $-1.62 \mathrm{~h})$ and FUP $(-0.91 \mathrm{~h})$ parasites.

Differentially expressed transcripts identified in HbAS in vitro. HbAS impacts $P$. falciparum's transcriptional program in the IDC at various time points in 3D7 and FUP isolates (Fig. 3A and B), though in both strains we observed an absence of differential expression through much of the ring stage, with transcriptional divergence apparent 
A
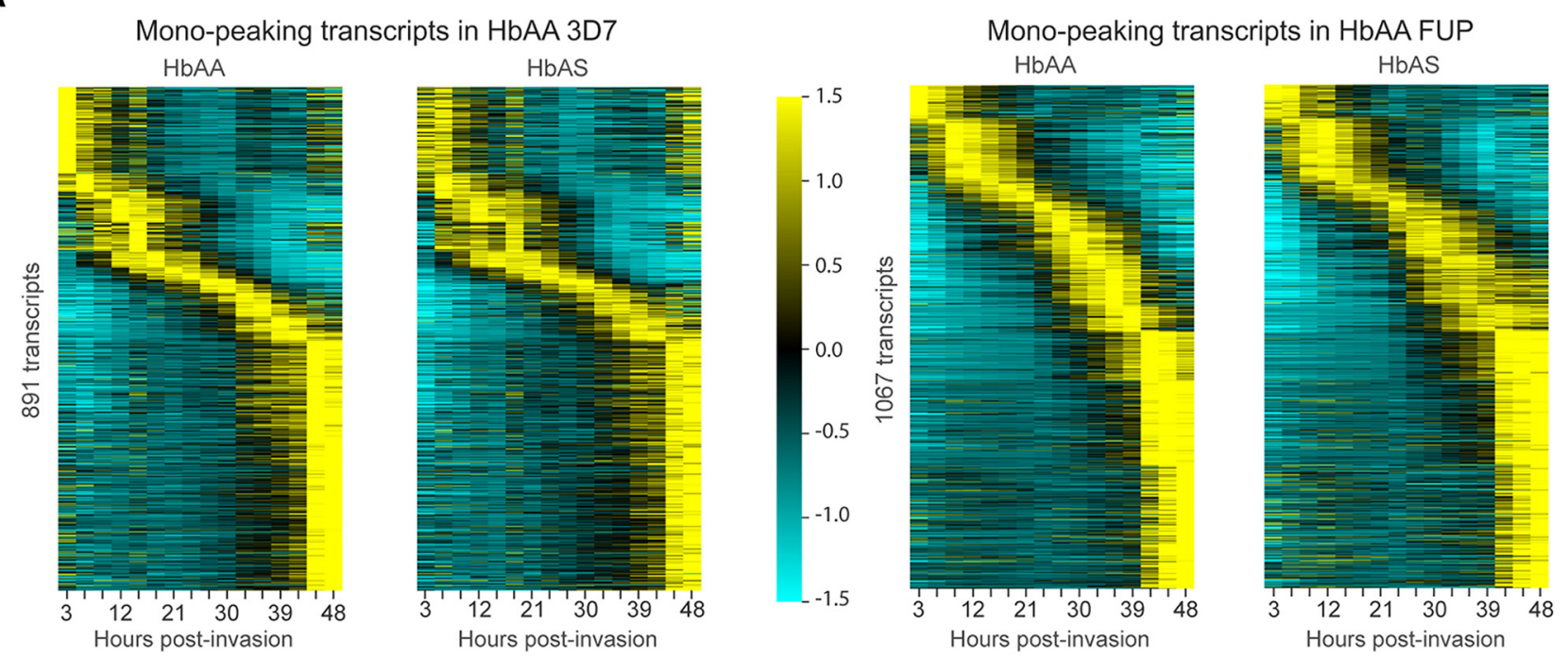

B

Shifts in peak transcription time between mono-peaking transcripts in HbAA and $\mathrm{HbAS}-3 \mathrm{D} 7$

Shifts in peak transcription time between mono-peaking transcripts in HbAA and HbAS - FUP
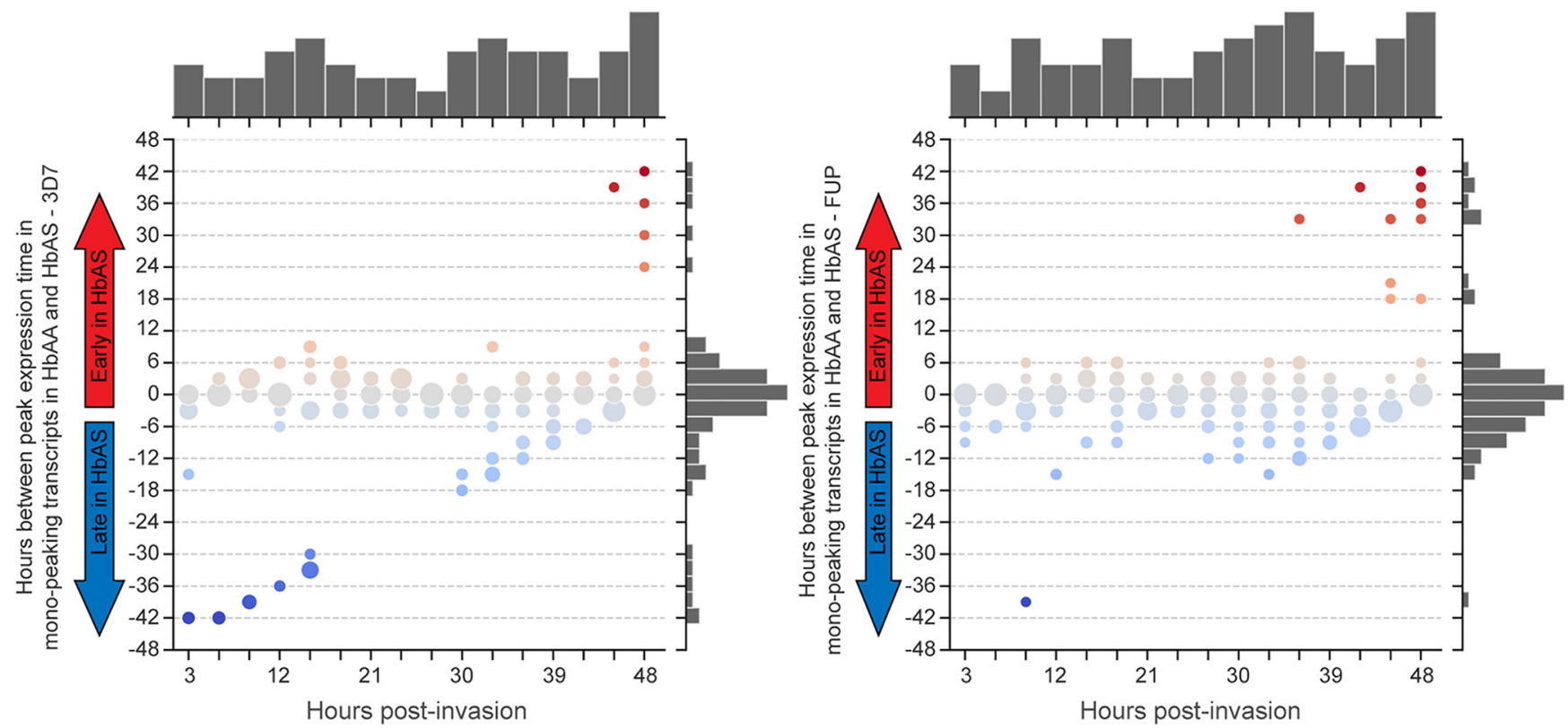

FIG 2 Shifts in peak expression time between $\mathrm{HbAA}$ and $\mathrm{HbAS}$. (A) Peak expression time of the subsets of mono-peaking parasite transcripts in HbAA and HbAS iRBCs for $3 D 7$ (left pair) or FUP (right pair) parasites. For each pair, transcripts are ordered vertically by the peak expression time in HbAA iRBCs. In both 3D7 and FUP, the ordering of expression is largely conserved between HbAA and HbAS samples. The columns along the $x$ axis depict the normalized expression values of each transcript per time point. Expression values were averaged across replicates and are normalized around the mean for each transcript over the time series and depicted as a $z$ score of standard deviations from the mean. Values range between -1.5 (cyan) and 1.5 (yellow). (B) Temporal shifts for peak expression time point for the subset of mono-peaking parasite transcripts in HbAA compared with HbAS iRBCs for 3D7 (left) or FUP (right) parasites. For each transcript, the time between its expression peak in parasites in HbAA and HbAS iRBCs was calculated and averaged across $\beta$-globin replicates for each strain. Each $x$-axis category indicates the number of transcripts that normally peak at this time point in HbAA iRBCs, and the $y$ values indicate the distributions of the shifts of the peaks of these transcripts in HbAS iRBCs. Circles are sized relative to the number of transcripts with that $y$ value. The top histogram indicates the number of parasite transcripts that peak in HbAA iRBCs at each $x$-value time point, and the right histogram indicates the number of transcripts with peak shifts of each $y$ value. The distribution of peak shifts (displayed along the right $y$ axis) is centered around zero, indicating that most transcripts peak at the same time in parasites growing in HbAA and HbAS iRBCs. See Jupyter Notebook "expressionPeakChanges.ipynb" on our GitHub for the full workflow.

in the mid- to late-trophozoite stage that accelerated as parasites entered the schizont stage (Fig. 3B).

We identified the transcripts that were differentially expressed in the same direction in both 3D7 and FUP at each time point (Fig. 3A), which indicated a consistent late-stage effect of HbAS on transcriptional phenotypes across parasite strains (Fig. 3B). In a gene 
A

Adjusted $\mathrm{p}$-value

$p p>0.05 \bigcirc 3 D 7: p<0.05 \quad$ FUP: $p<0.05$

3D7 \& FUP: $p<0.05$

3 hours post-invasion

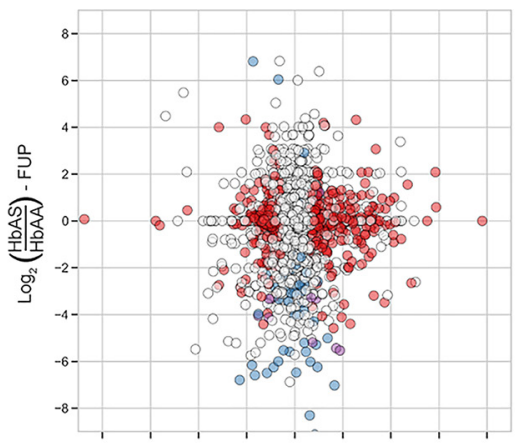

36 hours post-invasion

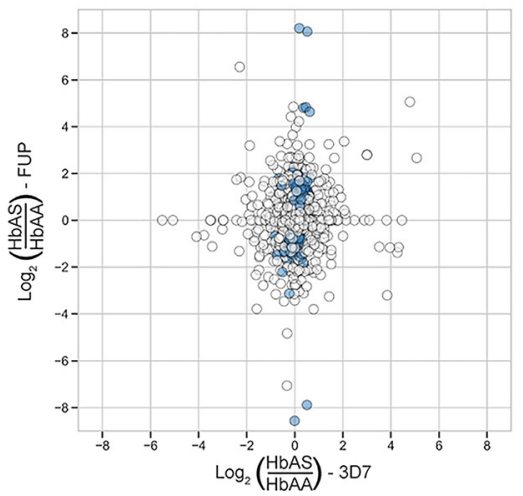

B

Number of transcripts Number of transcripts down-regulated in $\mathrm{HbAS}$ up-regulated in $\mathrm{HbAS}$
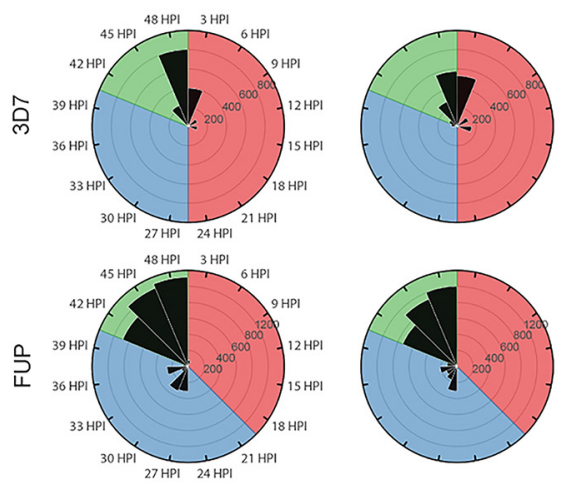

Ring

Trophozoite

Schizont
12 hours post-invasion

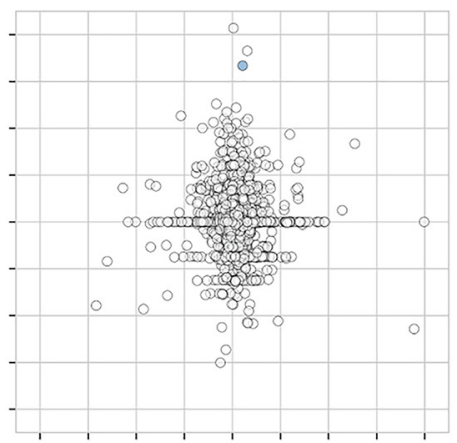

42 hours post-invasion

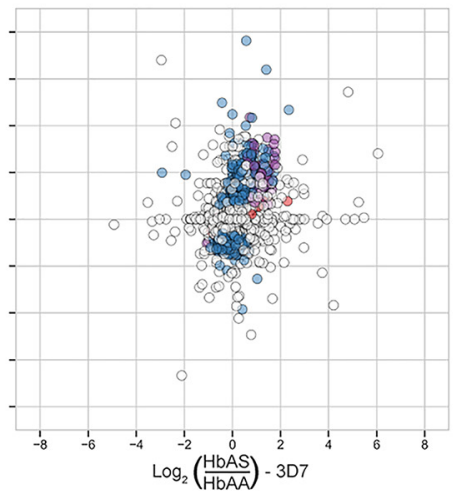

C

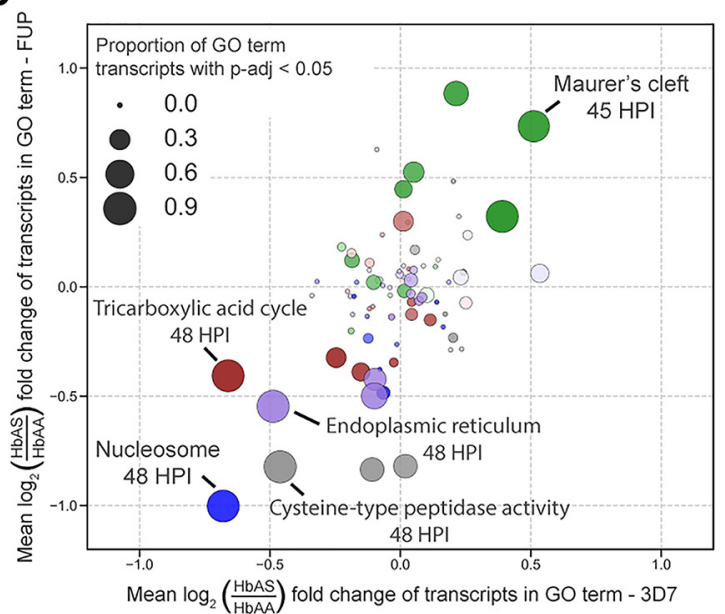

24 hours post-invasion

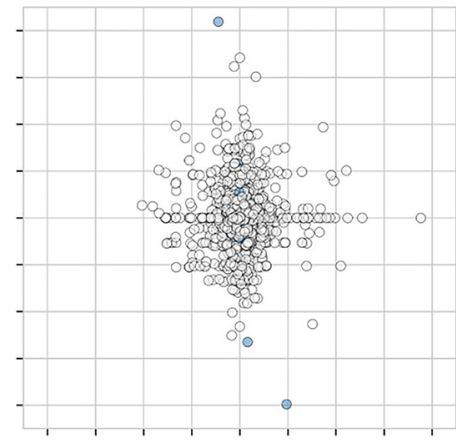

48 hours post-invasion

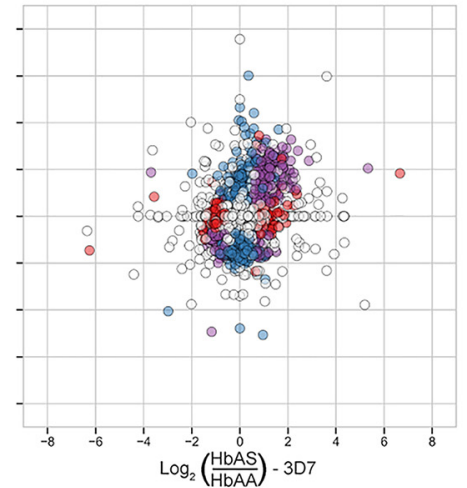

GO term

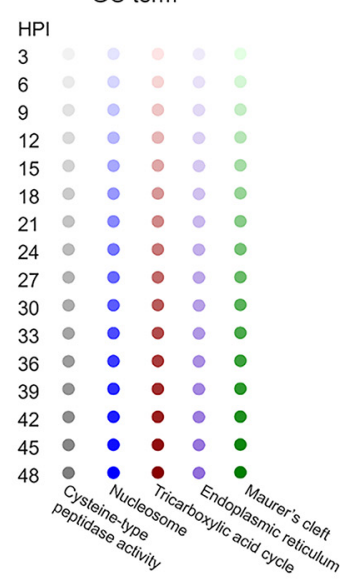

FIG 3 Differential expression analysis reveals parasite transcript expression in HbAS iRBCs diverges from HbAA parasites primarily in the schizont stage. (A) Scatterplots at $3,12,24,36,42$, and $48 \mathrm{~h}$ postinvasion of the $\log _{2}$ fold change of individual transcripts for HbAS parasites compared to $\mathrm{HbAA}$ parasites (hpi) in $3 \mathrm{D} 7$ ( $x$ axes) and FUP ( $y$ axes) parasites. Points in the upper right and lower left quadrants signify transcripts that are differentially expressed in the same direction between parasite strains, i.e., those in the upper right quadrant are upregulated in both 3D7 and FUP in HbAS iRBCs, and those in the lower left quadrant are downregulated in both 3D7 and FUP in HbAS iRBCs. Point color indicates the statistical significance of DESeq2 differential expression analysis for each transcript: white points have an adjusted $P$ value of $>0.05$ in both 3D7 and FUP, red points have an adjusted $P$ value of $<0.05$ in 3D7 but not FUP, blue points have an adjusted $P$ value of $<0.05$ in FUP but not 3D7, and purple points have an adjusted $P$ value of $<0.05$ in both $3 D 7$ and FUP. (B) Black bars indicate counts of transcripts in 3D7 (top) and FUP (bottom) classified as downregulated (left column) or upregulated (right column) at a significance level of an adjusted $P$ value of $<0.05$ at each time point, from $3 \mathrm{hpi}$ to $48 \mathrm{hpi}$. Each plot is colored according to the predominant stage of the parasites at each time point: ring (red), trophozoite (blue), or schizont (green). There were minimal shared differentially expressed transcripts until 39 hpi, after which 966 transcripts were differentially expressed in both 3D7 and FUP (493 downregulated and 460 upregulated). (C) Scatterplot of transcript mean fold changes within gene ontology (GO) terms that are enriched for differentially expressed transcripts in HbAS iRBCs in both 3D7 ( $x$ axis) and FUP ( $y$ axis) parasites. Color indicates GO term, shading indicates hpi (darker = later $\mathrm{hpi}$ ), and size of point indicates the proportion of transcripts within the GO term that are differentially expressed at an adjusted $P$ value of $<0.05$. Significant expression changes of GO terms occurred at later time points during the schizont stage, including upregulation of Maurer's cleft transcripts in HbAS iRBCs and concurrent downregulation of transcripts involved in the endoplasmic reticulum, the tricarboxylic acid cycle, cysteine-type peptidase activity, and nucleosomes. 
A

Log2 fold change of Maurer's cleft transcripts
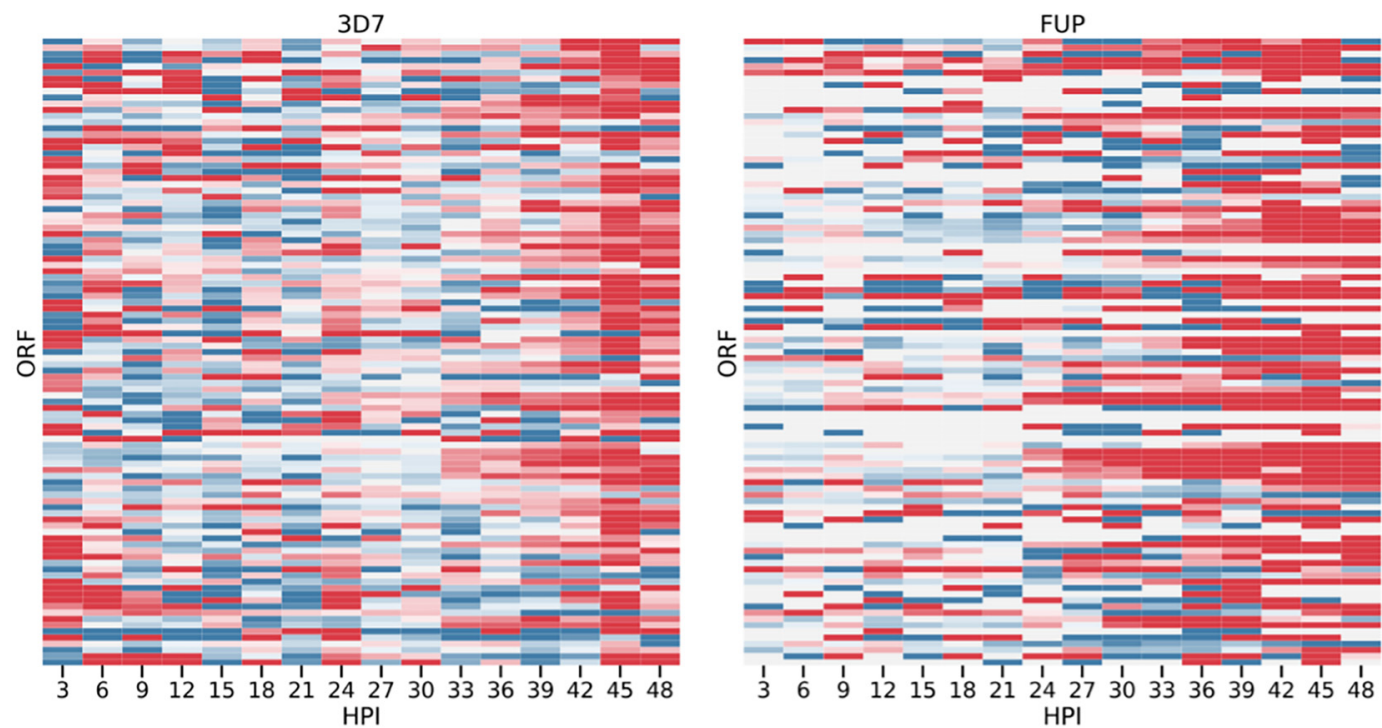

B

Log2 fold change of histone transcripts
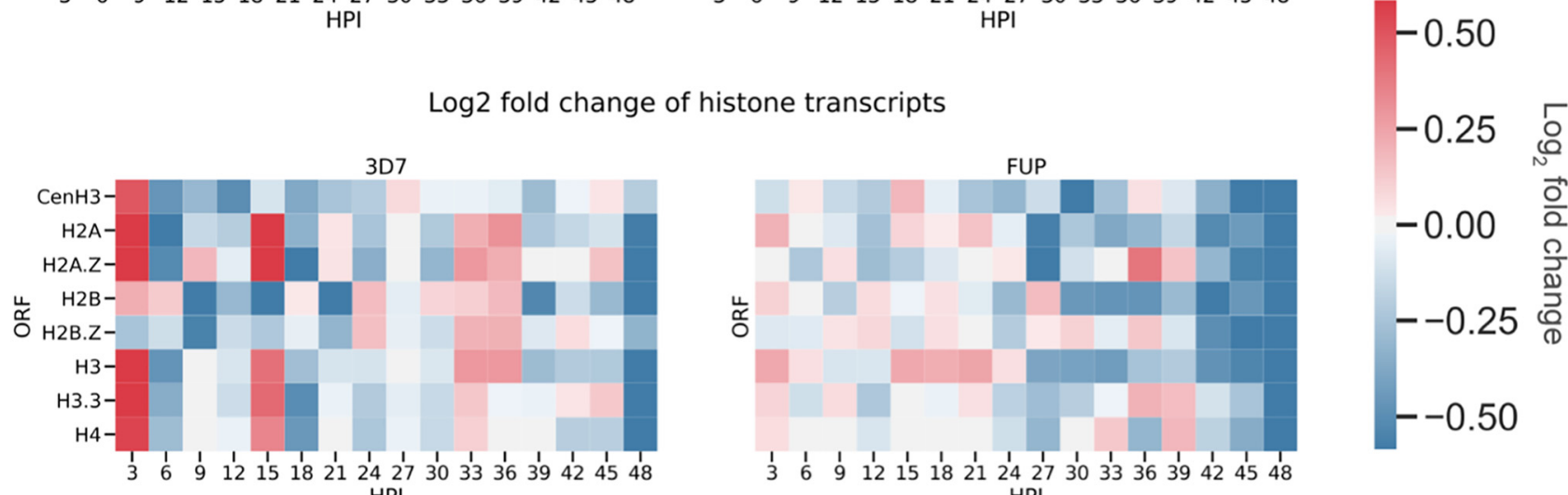

$\mathrm{HP}$

C

Log2 fold change of SERA transcripts
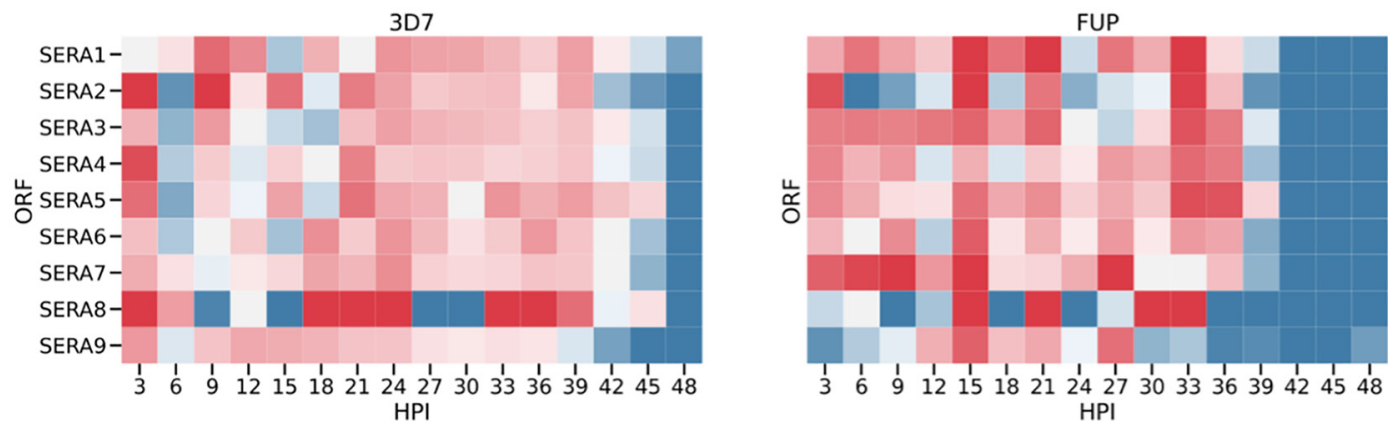

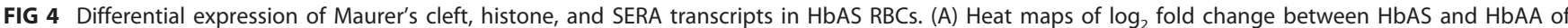
Maurer's cleft transcripts in 3D7 and FUP parasites. Transcript IDs were extracted from the Maurer's cleft GO term (GO:0020036). (B) Heat maps of log ${ }_{2}$ fold change between $\mathrm{HbAS}$ and $\mathrm{HbAA}$ of histone transcripts in 3D7 and FUP parasites. Transcript IDs were extracted from the nucleosome GO term (GO:0000786). (C) Heat maps of $\log _{2}$ fold change between HbAS and HbAA of SERA transcripts in 3D7 and FUP parasites. The color bar is restricted to log 2 fold change considered significant in this study $\left[\left|\log _{2}(\mathrm{HbAS} / \mathrm{HbAA})\right|>\log _{2}(1.5)\right]$.

ontology (GO) enrichment analysis of this shared set of transcripts (17) (Fig. 3C), the only GO term that was enriched for upregulated transcripts in both 3D7 and FUP in HbAS iRBCs at any time point in our time series experiments was that for Maurer's clefts (Fig. 4A), which are parasite-derived membranous structures that sort and traffic proteins 
and in HbAS iRBCs are both dysmorphic and dysfunctional (8). Several critical components of Maurer's clefts, including the membrane-associated histidine-rich protein 1 (MAHRP1; PF3D7_1370300), and skeleton-binding protein 1 (SBP1; PF3D7_0501300), are encoded by transcripts that were among the most significantly upregulated in HbAS RBCs.

The most downregulated GO term in both 3D7 and FUP in HbAS iRBCs was that of the nucleosome (GO:0000786), which comprises eight transcripts that encode the core histones and histone variants in P. falciparum. In both 3D7 and FUP growing in HbAS RBCs, all histone transcripts were downregulated at 48 hpi (Fig. 4B). Similarly, downregulated transcripts were enriched in the tricarboxylic acid (TCA) cycle (GO:0006099) and the endoplasmic reticulum (ER) (GO:0005783) at late time points in 3D7 and FUP in $\mathrm{HbAS}$ iRBCs. The ER GO term encompasses a wide range of proteins, including heat shock proteins, phosphatases, peptidyl-prolyl cis-trans-isomerases, and plasmepsins. Of 53 transcripts in the ER GO term, 30 transcripts were downregulated in both strains in $\mathrm{HbAS}$ iRBCs between 45 and $48 \mathrm{hpi}$.

Among molecular function GO terms, downregulated transcripts were enriched only in that of cysteine-type peptidase activity (GO:0008234), which primarily comprises the serine repeat antigen (SERA) proteases that play a pivotal role enabling parasite egress from the iRBC. SERA2 to -7 were significantly downregulated in 3D7 and FUP parasites in HbAS iRBCs at late time points (Fig. 4C).

Temporal dysregulation of transcript expression in HbAS iRBCs. To identify the transcripts that are temporally dysregulated, we analyzed our time series data using dynamic time warping (DTW) (Fig. 5A), which determines between two series the optimal alignment of a transcript's expression and assigns a score as a measure of similarity (low score) (Fig. 5B, top row) or difference (high score) (Fig. 5B, bottom row). Overall, DTW scores comparing transcript temporal expression between $\mathrm{HbAA}$ and $\mathrm{HbAS}$ RBCs were close to normally distributed with a positive skew in 3D7 and in FUP (Fig. 5A). Between strains, mean transcript DTW scores comparing $\mathrm{HbAA}$ versus $\mathrm{HbAS}$ time series were positively linearly correlated when computed for 3D7 or FUP $(r=0.524 ; P<0.0001)$, indicating a conserved impact on transcript timing across strains.

We observed in HbAS iRBCs 155 temporally dysregulated transcripts in 3D7 and 162 in FUP (Fig. 5C). In contrast to the generally unperturbed expression patterns of the overall transcriptome (Fig. 1C), these subsets of transcripts demonstrate aberrant expression profiles in HbAS iRBCs (Fig. 5C). Transcripts were grouped by GO terms and, for each term, the mean DTW score was calculated between parasites in HbAA and $\mathrm{HbAS}$. We observed that transcripts associated with antigenic variation and cytoadhesion (i.e., the variant surface antigens) were among the most conserved in the timing of their expression (Fig. 5D). In contrast, the highest DTW scores were recorded for transcripts involved in transcription by RNA polymerase II (GO:0006366), RNA splicing (GO:0008380), regulation of translational initiation (GO:0006446), and ribosome biogenesis (GO:0042254), which collectively suggest that HbAS may have posttranscriptional consequences beyond what can be observed in our data.

We focused more closely on ring-stage expression by screening with the temporal alignment Kendall-Tau (TAKT) algorithm, which measures the overall similarity and temporal shift between two curves (18), and then computing for these candidate transcripts DTW scores. Using this approach in ring-stage parasites, we identified 9 transcripts in 3D7 and 11 in FUP that were dysregulated between parasites in HbAA and $\mathrm{HbAS}$, though none of these were shared between the two strains.

In vivo transcriptomes of $\boldsymbol{P}$. falciparum in children with HbAS. We next compared parasite transcriptomes between 16 uncomplicated $P$. falciparum episodes in HbAS children matched to 16 episodes in HbAA children in Kéniéroba, Mali. We first used stage-specific ring ( $n=7$ transcripts), trophozoite $(n=4)$, and schizont $(n=6)$ transcripts to classify 24 infections as ring stage and 12 as trophozoite stage (Fig. 6A). PCA and hierarchical clustering supported these classifications (Fig. 6B). Late-stage trophozoites are rare in circulation (19), so we re-estimated the stage of each infection using an independent published mixture model, which suggested that the trophozoite classifications were early trophozoites (20) and otherwise confirmed initial findings. 
A

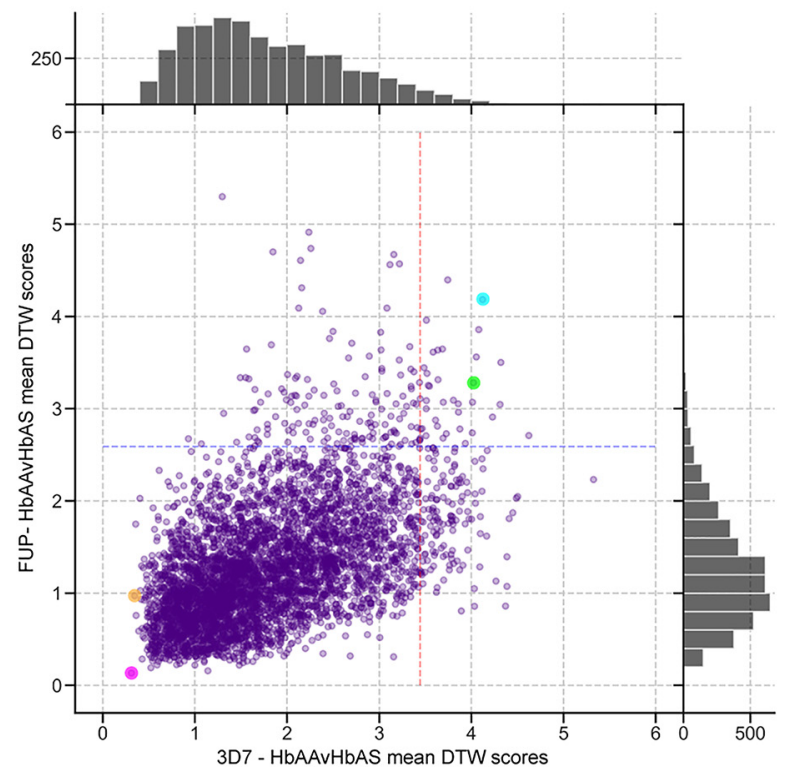

C
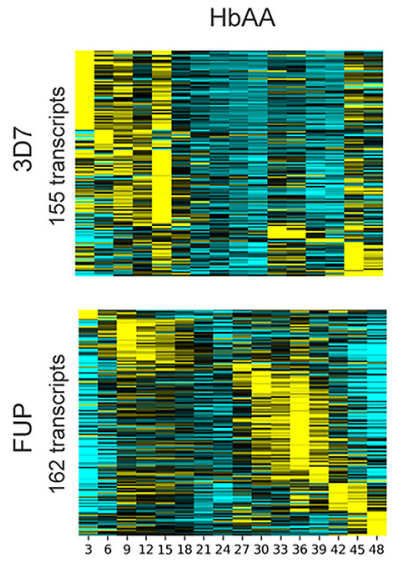

B

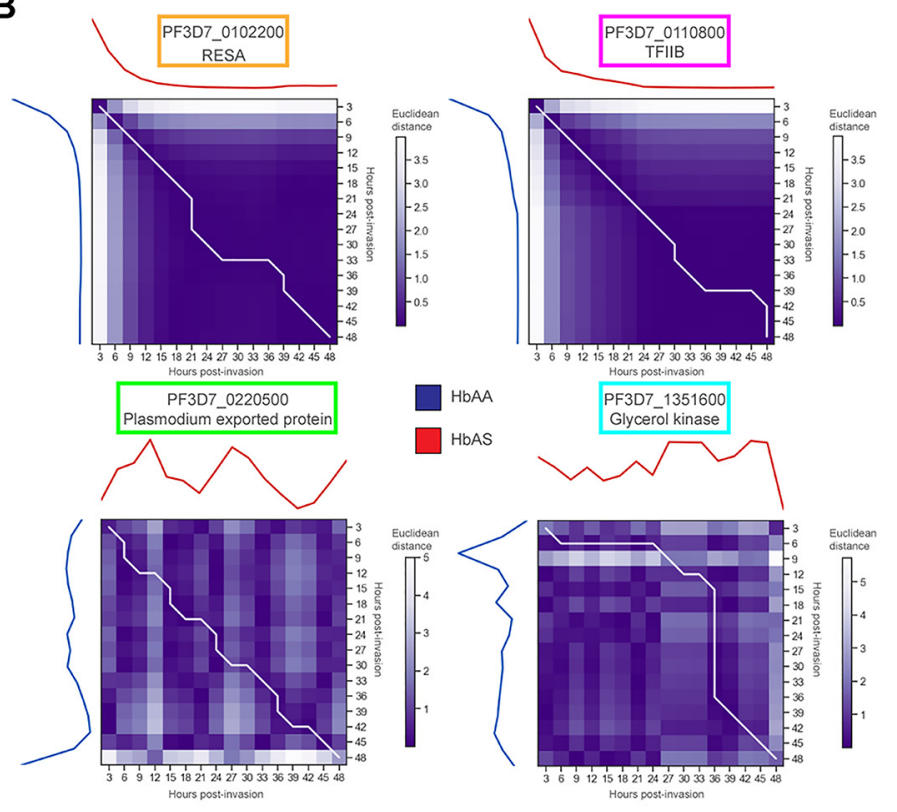

D

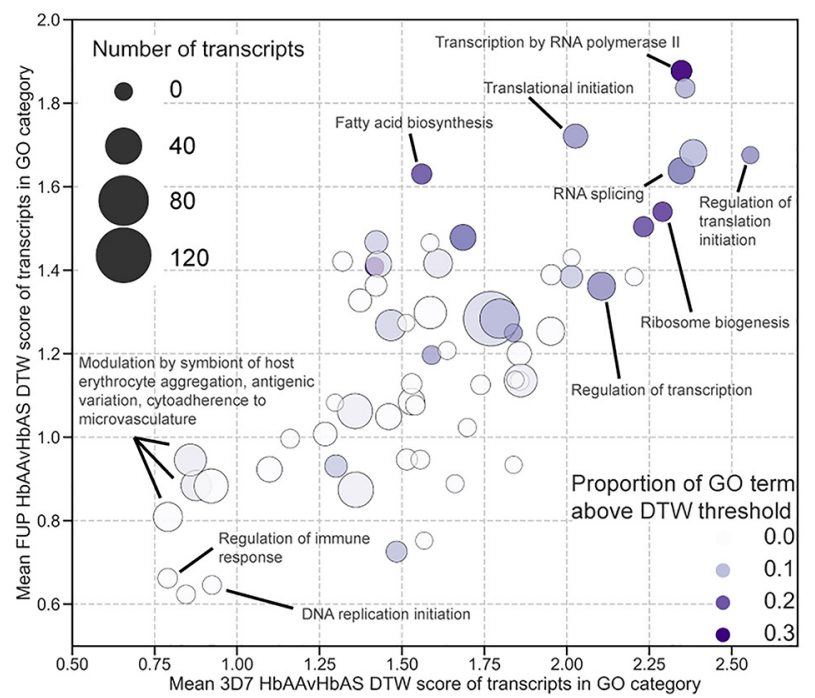

FIG 5 HbAS impacts the temporal expression of transcripts governing gene regulation. (A) Scatterplot of dynamic time warping (DTW) scores in time series data of individual transcripts in HbAA compared to HbAS samples in 3D7 ( $x$ axis) and FUP ( $y$ axis) parasites. For each strain, scores were computed pairwise between $\mathrm{HbAA}$ and $\mathrm{HbAS}$ samples and averaged. Transcripts with dynamic time warping scores greater than two standard deviations from the overall mean (points to the right of the red line for 3D7 and points above the blue line for FUP) were considered temporally dysregulated. Histograms indicate overall distribution of transcript DTW scores in either 3D7 (top) or FUP (right). (B) The Euclidean distance scores and warping path of selected transcripts that correspond to the highlighted circles in panel A. Mean-normalized transcript expression is plotted along the $x$ and $y$ axes: that for HbAA iRBCs is plotted from top to bottom along the left $y$ axis, and that for HbAS iRBCs is plotted from left to right along the upper $x$ axis. The heat map matrix is colored according to the Euclidean distance between the mean-normalized expression values between HbAA and HbAS at the corresponding time points. Dynamic time warping scores are cumulatively computed along the warping path (white line). Transcripts for the ring-infected erythrocyte surface antigen (RESA, PF3D7_010220) and the putative transcription factor TFIIB (PF3D7_0110800) (top) had very low DTW scores between HbAA and HbAS samples in both strains, while those for the Plasmodium exported protein (hyp2; PF3D7_0220500) and a glycerol kinase (PF3D7_1351600) had high DTW scores, indicating dissimilar transcriptional profiles between HbAA and HbAS RBCs (bottom). (C) Heat maps of mean-normalized expression of the 155 (3D7; top) and 162 (FUP; bottom) transcripts with DTW scores exceeding two standard deviations from the mean score in HbAA (left) and HbAS (right) iRBCs (points beyond the dashed lines in panel A). Transcripts for each parasite strain are order vertically by their peak expression time in HbAA iRBCs. The ordering of peak expression in HbAS samples is not conserved in temporally dysregulated transcripts identified by DTW. Transcripts are ordered by peak expression time in HbAA samples and displayed as a $z$ score of standard deviations from the mean. (D) Scatterplot of mean DTW scores within GO terms that are enriched for temporally dysregulated transcripts in HbAS iRBCs in both 3D7 ( $x$ axis) and FUP ( $y$ axis) parasites. Color indicates GO term, shading indicates hpi (darker = later hpi), and size of point indicates the proportion of transcripts within the GO term that are temporally dysregulated as defined by a DTW score greater than 2 standard deviations from the overall mean of DTW scores (points beyond the dashed lines in panel A). Transcripts involved in gene regulation processes demonstrate higher DTW scores. See Jupyter Notebook "DTW.ipynb" on our GitHub for full workflow. 

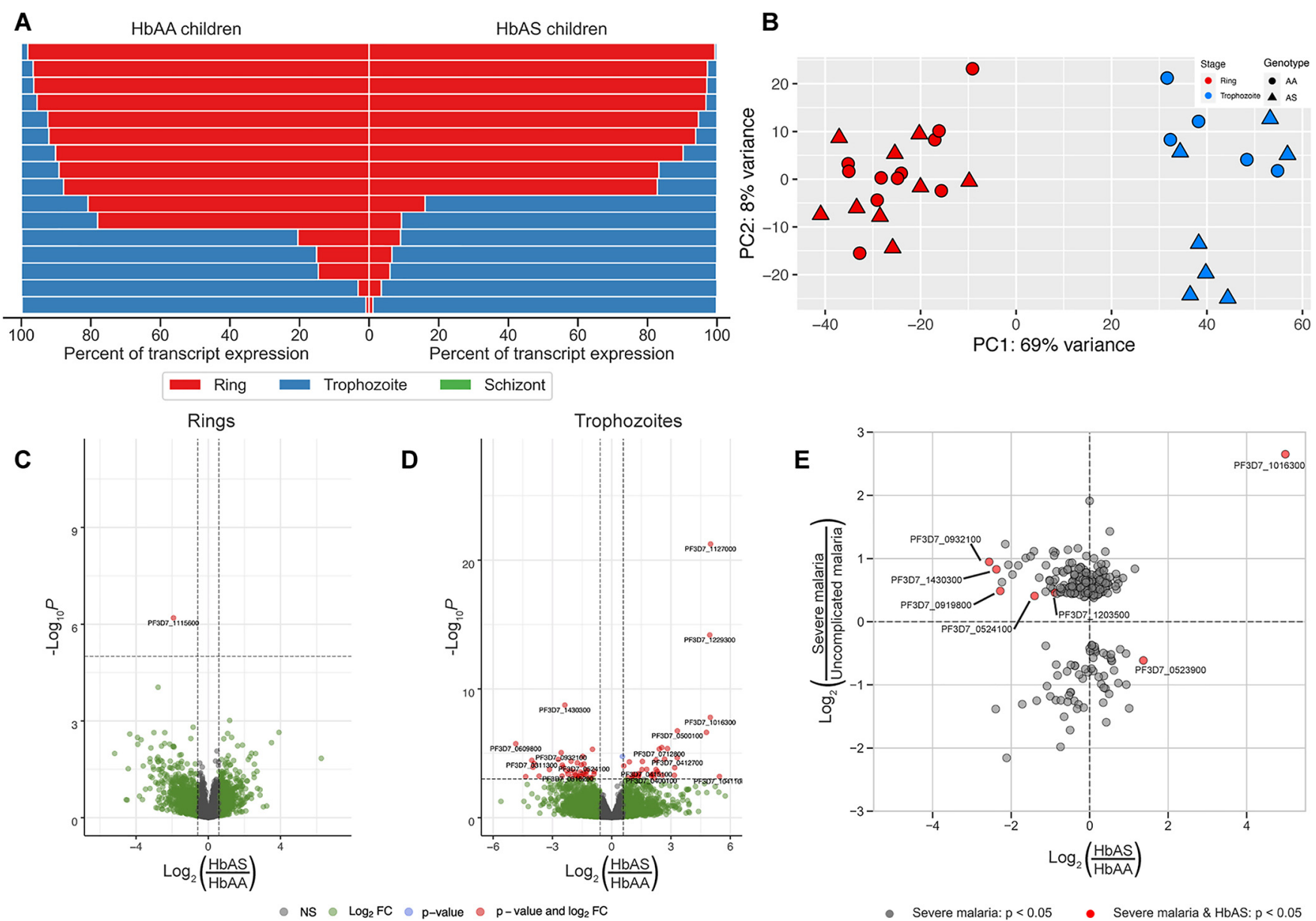

FIG 6 Differential expression profiling of parasite transcripts in freshly collected infections in Malian children with uncomplicated malaria with HbAA or HbAS. (A) Proportion of parasite stage-specific transcripts expressed in each patient's infection (rows) in HbAA (left) and HbAS (right) children. Expression of stage-restricted transcripts for ring (red), trophozoite (blue), and schizont (green) stages were measured as TPM, summed within each stage, and expressed as a proportion of stage for each infection. We observed a nearly complete absence of schizont-specific transcript expression, and most infections were predominantly either rings or trophozoites with little admixture. See Jupyter Notebook "Genes_by_stage.ipynb" on our GitHub for the full workflow. (B) Principal-component analysis of transcript expression from in vivo isolates following variance-stabilizing transformation based on the 500 transcripts with the highest variance across all samples. Clusters separate according to parasite stage identified from stage-specific transcript expression. (C and D) Volcano plots of differential transcript expression conditioned by parasite stage. The $x$ axis shows the log fold change of HbAS versus HbAA children, with positive values indicating increased expression in HbAS. The $y$ axis shows $-\log _{10} P$ values of each transcript, and horizontal dashed lines indicate $P$ value thresholds that capture the transcripts with adjusted $P$ values of $<0.05$. (E) Scatterplots of the $\log _{2}$ fold change of individual transcripts for severe-malaria parasites compared to uncomplicated-malaria parasites in Gambian children ( $y$ axes) and HbAS compared to HbAA parasites ( $x$ axes). Points in the upper right and lower left quadrants signify transcripts that are differentially expressed in the same direction between studies; i.e., those in the upper right quadrant are upregulated in both severe malaria and HbAS, and those in the lower left quadrant are downregulated in both severe malaria and $\mathrm{HbAS}$. All transcripts included were statistically significant in severe malaria versus uncomplicated malaria (26). Red points have an adjusted $P$ value of $<0.05$ in trophozoite parasites from $\mathrm{HbAS}$ versus $\mathrm{HbAA}$ Malian children.

Differential expression in vivo. The only differentially expressed transcript in ringstage infections was cyclophilin 19B (cyp19B; PF3D7_1115600), a peptidyl-prolyl cistrans-isomerase (PPlase) (Fig. 6C): compared to children with HbAA, cyp19B in those with $\mathrm{HbAS}$ was downregulated 3-fold (adjusted $P=0.023010$ ). Quantitative PCR (qPCR) confirmed that cyp19B is reduced in ring-stage parasite transcriptomes in HbAS. Cyclophilins function in protein folding and trafficking, in RNA processing, and generally as molecular chaperones (21) and are most highly expressed in schizont-stage parasites in vitro (22).

We observed in trophozoite-stage patient samples differential expression of 74 transcripts (Fig. 6D), among which we did not observe any enrichment for GO terms. The most upregulated transcript in $\mathrm{HbAS}$ trophozoites was a putative protein phosphatase (PF3D7_1127000; $\log _{2}$ fold change $=5.00$; adjusted $P=1.08 \times 10^{-18}$ ). Also among the 10 most upregulated transcripts in $\mathrm{HbAS}$ trophozoites were the gene for the $130-\mathrm{kDa}$ 
glycophorin binding protein (GBP130; PF3D7_1016300) and the two glycophorin binding protein homologs (GBPH, PF3D7_1401000; GBPH2, PF3D7_1301200), each upregulated more than 8-fold in HbAS trophozoites. Additionally, one of the most significantly upregulated transcripts in HbAS trophozoites ( $\log _{2}$ fold change $=1.55$; adjusted $P=0.0077$ ) encodes the heat shock protein 70-x (HSP70-x; PF3D7_08317000), which is thought to chaperone export of PfEMP-1 via J-dots and Maurer's clefts (23), similar to the 2-fold overexpression of the critical Maurer's cleft components MAHRP1 (PF3D7_1370300; adjusted $P=0.041$ ) and MAHRP2 (PF3D7_1353200, adjusted $P=0.034$ ).

Among the 41 downregulated transcripts in trophozoites in HbAS children, the most highly significant was that encoding a putative acid phosphatase (PF3D7_1430300; $\log _{2}$ fold change $=-2.37$; adjusted $P=1.77 \times 10^{-6}$ ). We also observed among the top 10 downregulated transcripts the genes for putative autophagy protein 5 (ATG5) (PF3D7_1430400; adjusted $P=0.0076$ ), which associates with autophagosome-like structures (24), and for the DNA repair protein RAD5 (PF3D7_1343400; adjusted $P=0.0091)$, which has been linked to delayed clearance following artemisinin treatment (25).

Because HbAS confers protection specifically from severe malaria, we compared our data with those from a prior study comparing parasite gene expression in severe and uncomplicated malaria (26), which identified 236 differentially expressed transcripts. Seven of these transcripts were also differentially expressed between trophozoite-stage parasites in $\mathrm{HbAS}$ and $\mathrm{HbAA}$ children (Fig. 6E). Of these seven, six were transcripts overexpressed in severe malaria. One of these, gbp130 (PF3D7_1016300), was upregulated in children with $\mathrm{HbAS}$. Five of the six transcripts that were upregulated in severe malaria were downregulated in children with HbAS (PF3D7_0932100, PF3D7_1203500, PF3D7_0524100, PF3D7_0919800, and PF3D7_1430300). Among the transcripts with statistically significant upregulation in severe malaria but downregulation in HbAS RBCs was that of a putative acid phosphatase (PF3D7_1430300), which was the most statistically significantly downregulated transcript in our HbAS trophozoite samples compared to HbAA trophozoites.

Intersection of aberrant transcripts in HbAS across analytic approaches. Across analyses of both differential expression and temporal dysregulation in our time series experiments, only 15 candidate transcripts were aberrantly expressed in HbAS by 3D7 and FUP parasites (Fig. 7A). These include PTEX88, which was upregulated in both 3D7 and FUP, which is noteworthy owing to its critical role in exporting proteins from the parasitophorous vacuole membrane (PVM) that remodel the iRBC. Of these candidates, 10 overlapped candidates identified from in vivo infections in the same direction. The sole transcript differentially expressed in in vivo ring-stage parasites, cyp19B, was also downregulated in both 3D7 and FUP in HbAS (Fig. 7B to D).

Among the genes that were significantly upregulated in vitro and in vivo was gbp130, whose expression was increased more than 30-fold in HbAS trophozoites compared to HbAA. In our in vitro time series, we found that this gene is significantly upregulated in $\mathrm{HbAS}$ FUP at $48 \mathrm{hpi}$, and one HbAS 3D7 replicate also demonstrated upregulation at 48 hpi. In addition, the glycophorin-binding protein homologs, gbph and $g b p h 2$, were significantly upregulated in vivo in trophozoite-stage parasite isolates and in vitro in both HbAS 3D7 and HbAS FUP. In vitro, hsp70-x (PF3D7_0831700) demonstrated a pattern of elevated transcript abundance at late time points in 3D7 and FUP, although statistical significance was achieved only in FUP (Fig. 7C and D). Additionally, mahrp1 and mahrp2 were upregulated in $\mathrm{HbAS}$ in vitro and in vivo (Fig. $7 \mathrm{C}$ and D). Among downregulated transcripts in vivo and in vitro, we observed a palmitoyltransferase (PF3D7_0609800), a thiamine-phosphate pyrophosphorylase (PF3D7_0614000), an exported protein (hyp11, PF3D7_1102900) and two unannotated proteins of unknown function (PF3D7_0316200 and PF3D7_1449200). The protein phosphatase (PF3D7_1127000) that was the most highly upregulated transcript in HbAS trophozoites was highly expressed in HbAS and was also highly expressed and slightly upregulated in HbAS FUP, although it did not pass 
A

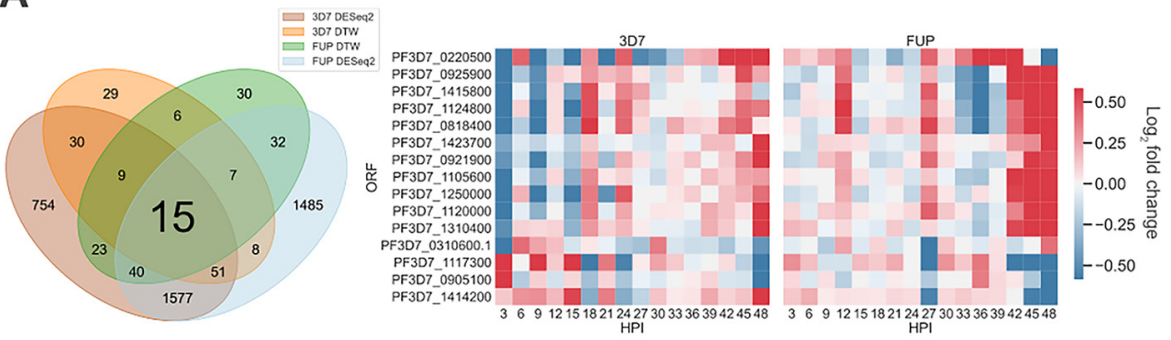

B

$\square$ in vitro upregulated
$\square$ in vivo upregulated
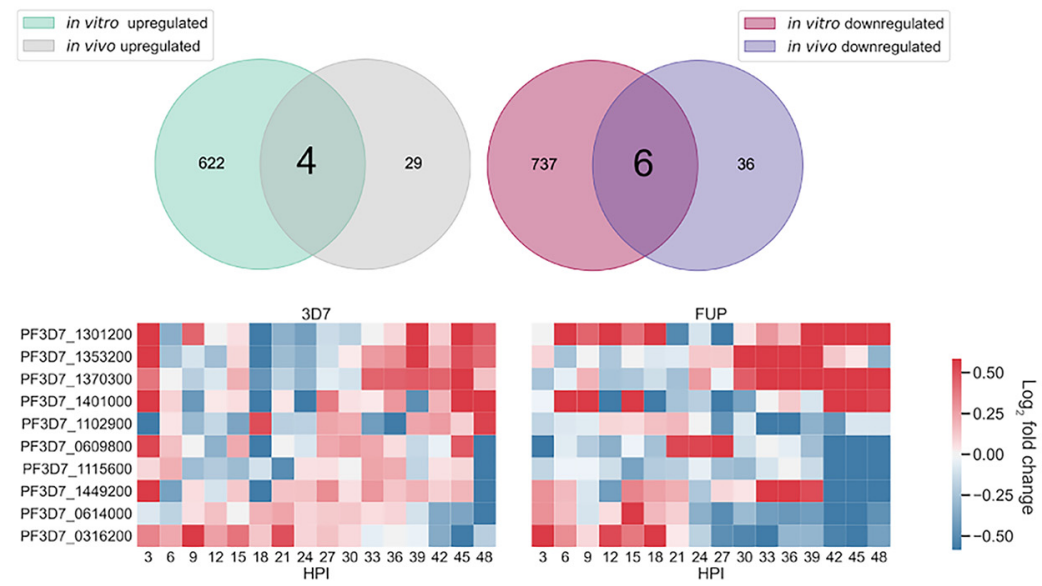

C
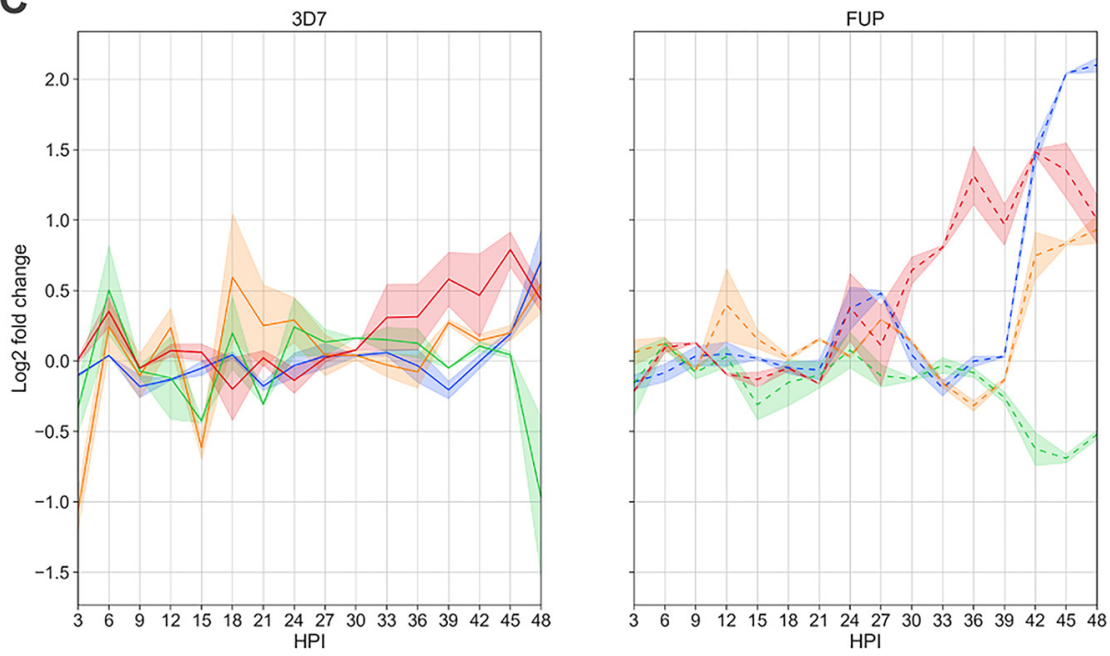

- HSP70-x

- PTEX88

CYP19B - MAHRP1
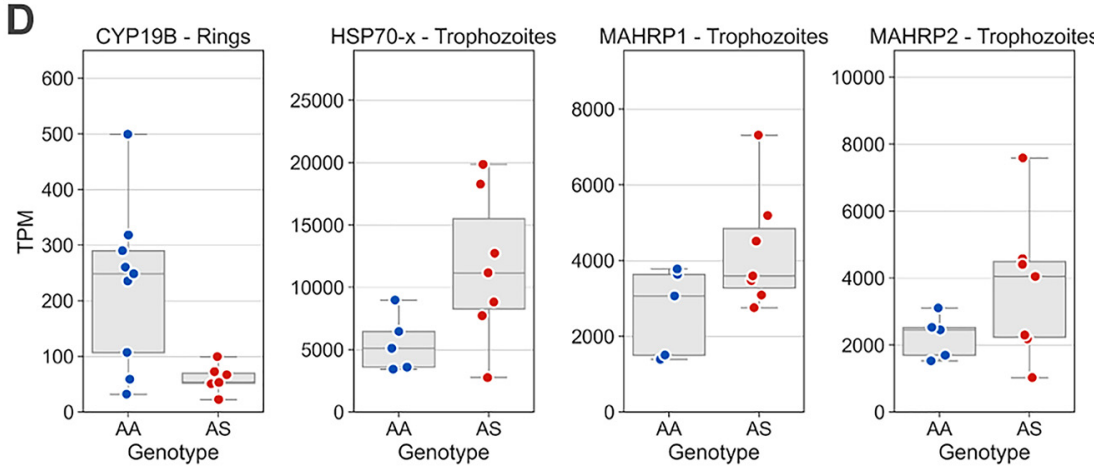

FIG 7 Intersection of aberrant transcripts across analytical and experimental approaches. (A) Venn diagram of transcripts that were differentially expressed and temporally dysregulated in 3D7 and FUP 
the threshold for statistical significance. In 3D7, however, this transcript was expressed at low levels in all samples.

Finally, we examined the set of candidate aberrant transcripts identified in vivo that were similarly expressed between $\mathrm{Hb}$ genotypes in vitro, a set which may indicate parasite responses that are specific to physiologic conditions. These 17 in vivo-specific transcripts include upregulation of atg5 (PF3D7_1430400) and rad5 (PF3D7_1343400), suggesting that autophagy and DNA repair, as well as the differential expression of enzymes whose specific functions are currently not well understood, including the putative protein phosphatase PF3D7_112700 and the putative acid phosphatase PF3D7_1430300, are impacted by HbAS in vivo.

\section{DISCUSSION}

HbAS consistently protects against severe $P$. falciparum malaria by mechanisms that remain obscure. In this study, we investigated the transcriptional changes that occur in $P$. falciparum in response to $\mathrm{HbAS}$, and overall, the parasite's transcriptional program remains largely unperturbed in HbAS iRBCs. However, hundreds of transcripts demonstrated differential expression in HbAS, with the bulk of these changes occurring late in the IDC. Combining our in vitro experiments with parasite transcript expression in vivo, we observed impacts of HbAS on transcripts encoding the parasite's protein chaperone and folding machinery, oxidative stress response, and protein export machinery.

Transcripts that were most clearly temporally dysregulated in HbAS iRBCs were those that are components of gene regulation: the GO biological processes with the highest DTW scores were those for transcription by RNA polymerase II, RNA splicing, regulation of translation initiation, and ribosome biogenesis (Fig. 5D). Mistimed expression of these transcripts could result in a cascade of downstream expression changes. In particular, temporal dysregulation of the subunits of RNA polymerase II, the complex responsible for the production of all parasite $\mathrm{mRNA}$, could result in altered transcript abundance. Furthermore, the downstream impact of temporal dysregulation of transcripts involved in RNA splicing, ribosome biogenesis, and translation initiation could lead to significant alterations in the HbAS parasite proteome. These potential posttranscriptional effects of $\mathrm{HbAS}$ on the parasite would be consonant with our parallel study of the effect of HbAS on the transcription of var genes and the presentation on the RBC surface of $P$. falciparum erythrocyte membrane protein 1 (PfEMP1) (27). In those experiments, HbAS allows var transcription but attenuates both the presentation of PfEMP1 on the RBC surface and the adhesion of infected RBCs to extracellular ligands, suggesting that posttranscriptional impairment of var translation or transport is an important component of attenuated pathogenesis.

Differential expression in HbAS emerged primarily during the schizont stage in our in vitro time series. The molecular functions and cellular components that were most downregulated in HbAS include cysteine-type peptidase activity, including the SERA proteins and the core histones and histone variants (Fig. $3 \mathrm{C}$ ). The SERA proteins play a role in erythrocyte rupture due to their localization to the parasitophorous vacuole (PV) in late stages of the IDC and to a papain-like cysteine peptidase domain that is

FIG 7 Legend (Continued)

in in vitro time series experiments. The 15 transcripts that were differentially expressed and temporally dysregulated in 3D7 and FUP are visualized in a heat map of $\log _{2}$ fold change between $\mathrm{HbAS}$ and $\mathrm{HbAA}$ samples over $48 \mathrm{~h}$. This list includes seven unannotated transcripts (PF3D7_0921900, PF3D7_1423700, PF3D7_1117300, PF3D7_1414200, PF3D7_0925900, PF3D7_1120000, and PF3D7_1310400), an exported protein (PF3D7_0220500), two rRNA processing proteins (PF3D7_1250000 and PF3D7_0818400), a translation initiation factor (PF3D7_0310600.1), a nuclear preribosomal assembly protein (PF3D7_1124800), a nucleoporin (PF3D7_0905100), a dimethyladenosine transferase (PF3D7_1415800), and the translocon component PTEX88 (PF3D7_1105600). (B) Venn diagram of transcripts that are up- and downregulated in both in vitro time series and in vivo samples from Malian children. The 10 transcripts that are shared between both experiments are visualized in a heat map, constrained to the conditions specified for panel A. (C) $\log _{2}$ fold change of transcripts over the in vitro time series of transcripts of biological significance that were included in panels A and B. (D) Normalized expression of transcripts of transcripts of biological significance in in vivo samples from panel $B$. 
common to all SERA proteins (28). The highly expressed SERA5 (29) and SERA6 are essential to blood-stage parasite growth $(30,31)$, and SERA5 inactivation disrupts schizont rupture and merozoite release (32). Disruption of individual SERA expression can result in compensatory overexpression of other SERAs (33), though our observation of consistent and significant downregulation of SERA2, -3, -4, -5, -6, and -7 (Fig. 4C) precludes this in $\mathrm{HbAS}$ and suggests that SERA-mediated egress is impaired in HbAS. IDC propagation also relies on a highly regulated epigenome (34), which is normally regulated by histones transcribed late in the asexual blood stage of development for nucleosome packaging in merozoites $(35,36)$. Transcripts encoding $P$. falciparum histones were consistently underexpressed in HbAS iRBCs (Fig. 4B), which could produce an altered chromatin landscape in the new merozoites and alter the blood stage transcriptional program after subsequent invasions. Though we did not observe large transcriptional changes in in vivo parasites from $\mathrm{HbAS}$ children, such an effect in vivo could be masked if such a defective transcriptional program inhibits these merozoites from successfully parasitizing an RBC. Taken together, these observations could collectively reduce the efficiency of propagation. With confirmation during consecutive in vitro cycles in HbAS RBCs of the abundance of SERA5 transcripts and protein, this could explain why HbAS is associated with lower parasite densities in vivo (37-39).

$\mathrm{HbAS}$ results in dysmorphic and dysfunctional Maurer's clefts (8, 40, 41). Surprisingly, we found that transcripts related to Maurer's clefts were among the most upregulated transcripts both in vitro and in vivo. $P$. falciparum exports proteins, including PfEMP-1, to the iRBC surface in knob-like protrusions that mediate its cytoadherence. Maurer's clefts serve as the hub through which these proteins are trafficked (42). In particular, sbp1, mahrp1, and mahrp2 were upregulated in both of our in vitro strains, with the latter two also upregulated in vivo in trophozoite-stage parasites (Fig. 7D). In the asexual blood stage, sbp 1 and mahrp 1 are nonessential, but individual disruption of either gene leads to dysmorphic Maurer's clefts and the absence of PfEMP-1 on the iRBC surface $(43,44)$. In the IDC, mahrp2 is essential, and the MAHRP2 protein localizes to electron-dense tubular structures known as tethers that connect Maurer's clefts to the parasitophorous vacuole membrane (PVM) and the RBC membrane $(45,46)$. These data suggest the possibility that gene expression of Maurer's clefts is under transcription-translation feedback control that is contingent upon the proper downstream assembly and function of its protein complexes. In this scenario, parasites in HbAS iRBCs would continue to express Maurer's cleft genes to compensate for the dysmorphic and dysfunctional Maurer's clefts.

Of particular interest among the six transcripts that were significantly downregulated in HbAS both in vitro and in vivo was cyp19B, which in our in vivo samples was approximately 3-fold downregulated. CYP19B localizes to the parasite cytosol and exhibits PPlase activity, participating in protein folding and potentially acting as a molecular chaperone via protein trafficking and regulation of multiprotein complexes (22). CYP19B is bound by cyclosporine (CsA), which inhibits in vitro both the PPlase activity of CYP19B (47) as well as the growth of P. falciparum (22). Interestingly, cyp19B was identified as the most upregulated gene in artemisinin-resistant (Art-R) parasites in a Southeast Asian population transcriptomic survey, in which its expression was the most positively correlated single transcript with increased parasite clearance half-life (48), where its upregulation correlated with a robust parasite stress response to the free-radical damage initiated by artemisinin. In contrast, in our experiments cyp $19 B$ was underexpressed in $\mathrm{HbAS}$ RBCs, despite the presumptively oxidizing and harsh environment of the HbAS RBC. CYP19B is also a member of the Plasmodium reactive oxidative stress complex (PROSC), and we observed a downregulation of a majority of the members of PROSC in HbAS in vitro at late time points in the IDC in both 3D7 and FUP, including cyp19B, hsp70-2, grp94, pdi8, and pferc, though not in vivo. Additionally, the relatively high levels of $c y p 19 B$ expression in $\mathrm{HbAA}$ in vivo ring-stage samples were unexpected, given that both transcript expression (in our in vitro time series) and protein expression (49) are highest in the late trophozoite and schizont stages. 
Among our in vivo parasites, parasites were either predominantly rings or trophozoites with no minor population of $>20 \%$, suggesting that circulating parasites in in vivo infections are broadly synchronized. This divergence accounted for most of the variance in transcriptomes, underscoring the need for stage-matched comparisons of parasite transcription. In vitro, most of the transcriptional changes we observed occurred in schizont-stage parasites. However, given that schizont-stage parasites are likely sequestered in the microvasculature and thus not in circulation, we could not confirm that similar changes occur in vivo. Finally, though we observed relatively few differentially expressed transcripts in HbAS children-particularly among the ring stages that predominated-the intersection of statistically significant differential expression between our trophozoite-stage data compared with transcriptomic data from severe malaria indicates that $\mathrm{HbAS}$ reduces the expression of a subset of transcripts that are associated with severe malaria. The functions of these transcripts are largely obscure, but our analyses collectively suggest their functional role in severe malaria.

Despite the expectation that HbAS would reduce microvascular cytoadherence and thereby produce more circulating trophozoites, we observed similar proportions of trophozoite-predominant infections in HbAS (7/16) and HbAA children (5/16) (Fig. 6A and $B$ ) and no evidence of late trophozoite or schizont gene expression in our $\mathrm{HbAS}$ patient samples (Fig. 6A). This may have been influenced by our sampling scheme, and since parasites are thought to be somewhat synchronous in natural infections in prior studies (50) and in our data, we would expect to find only ring-stage and early trophozoite parasites in our patients.

It is surprising that we observed conservation of the overall order of the transcriptional program in the IDC despite the presence of substantial temporal dysregulation in $\mathrm{HbAS}$ of gene regulatory GO terms such as RNA polymerase II and translation initiation. Interestingly, our in vitro time series data in HbAS RBCs mirrored key aspects of the transcriptional changes observed in $P$. falciparum lines with mutations of the drug resistance transporter genes pfmdr-1 and pfcrt (51), with an overall transcriptomic concordance followed by late-stage differential expression. This suggests the possibility that $P$. falciparum's transcriptional program in the IDC is sufficiently robust to adapt to fitness challenges which primarily manifest by modulating the expression levels of particular genes while still producing the necessary transcripts on time to complete the IDC. The maintenance of the parasite's transcriptional program relies on a robust architecture that is only partially understood, highlighted by recent reports that Plasmodium spp. possess intrinsic oscillators that govern the periodicity of their IDC program $(52,53)$. The alterations we observed in transcript abundances may be a consequence of the temporal dysregulation of gene regulatory products we discovered with DTW analysis. Such subtle alterations could, with successive rounds of infection, be amplified into fitness-mediating phenotypic changes. A need for successive rounds of infection to manifest transcriptional divergence in $\mathrm{HbAS}$ RBCs could also explain the paucity of overlap between the lists of differentially expressed transcripts in vitro and in vivo. Alternate explanations are the altered fitness landscape in the bloodstream of partially immune hosts, the sequestration in vivo of the most transcriptionally divergent schizont stages, or an interaction in vivo between $\mathrm{HbAS}$ and parasite genotype.

Our study had several limitations. Our in vitro time series experiments were conducted for one round of infection over $48 \mathrm{~h}$ in order to ensure tight synchrony of parasites, and therefore we could not observe impacts of perturbations in histones and chromatin modifications on subsequent infections. Our in vitro experiments were performed in single gas mixture at $1 \% \mathrm{O}_{2}$, while in vivo, $P$. falciparum encounters in circulation a range of oxygen tension that can differentially impact its growth in HbAS iRBCs in vitro (3-6). Though prior studies have reported a growth defect in HbAS RBCs at $1 \%$ $\mathrm{O}_{2}$, we did not observe such a defect over one IDC.

Our data encompass $P$. falciparum's transcriptional response to infection in $\mathrm{HbAS}$ RBCs, highlighting multiple avenues by which $\mathrm{HbAS}$ may neutralize $P$. falciparum. The 
potential neutralizing effect of these aberrations will require testing in systems of pathogenic phenotypes, including extracellular adhesion, endothelial activation, and immune evasion. These transcriptional perturbations induced in HbAS provide new targets to neutralize parasite mediators of disease and suggest that downstream changes in the proteome and epigenome are candidates for exploration.

\section{MATERIALS AND METHODS}

Subjects and samples. HbAA and HbAS blood samples were collected under institutional review board (IRB) number Pro00007816 for in vitro experiments. Genotypes were confirmed by Sanger sequencing. Field samples were collected in an observational study of the efficacy of artemether-lumefantrine for the treatment of uncomplicated malaria in children in Kéniéroba, Mali (ClinicalTrials.gov identifier NCT02645604), where HbAS reduces the risk of uncomplicated malaria by $34 \%$ (54). Inclusion criteria were age between 2 and 17 years and febrile uncomplicated falciparum malaria as assessed by light microscopy. From all participants, prior to treatment, we collected venous blood, passed this through cellulose columns (55), and stored up to $2 \mathrm{ml}$ of the flowthrough in RNAprotect (Qiagen). Parasite density was estimated from light microscopy, hemoglobin genotype was assessed using highperformance liquid chromatography (HPLC), and ABO blood group was assessed by agglutination assay. For transcriptional analysis, we selected all available samples from children with HbAS and matched each of these to a sample from a child with $\mathrm{HbAA}$ with regard to age (within 1 year), month of episode, parasite density, ethnic background, and, if possible, ABO blood type. The field study was approved by the Institutional Review Board of the University of Sciences, Techniques, and Technologies of Bamako (IRB00001983).

Parasite culture. $P$. falciparum parasites of the 3D7 and FUP strains were cultured in human RBCs in RPMI 1640 supplemented with Albumax II, L-glutamine, hypoxanthine, and D-glucose at a $2 \%$ hematocrit in $1 \% \mathrm{O}_{2}-5 \% \mathrm{CO}_{2}$ in $\mathrm{N}_{2}$ at $37^{\circ} \mathrm{C}$ (56). iRBCs were kept in filtered-lid cell culture flasks inside a modular hypoxia incubator chamber and mixed on a nutator. $P$. falciparum parasites from either strain were synchronized using Percoll centrifugation, and the schizont fraction was then split equally into separate flasks of HbAS or HbAA. Following a 3-h incubation, these cultures were treated with sorbitol and allowed to progress through the intraerythrocytic cycle in parallel, with sampling every $3 \mathrm{~h}$ for light microscopy and RNA preservation. Parasite density and maturation were assessed following Giemsa staining by readers masked to hemoglobin type and hours postinvasion.

Library preparation. Total RNA was isolated using TRIzol and the RNeasy minikit (Qiagen) after DNase treatment and quantified by a Qubit high-sensitivity RNA assay (Thermo Fisher Scientific) before storage at $-80^{\circ} \mathrm{C}$. Libraries were prepared from total RNA with the Kapa stranded mRNA-seq library prep kit. Libraries from each of the two time series experiments ( $n=64$ /experiment) were sequenced on a full flow cell on the NovaSeq 6000 S2 platform with 150-bp paired-end reads. Field study samples $(n=32)$ were sequenced on a full flow cell of the NovaSeq $6000 \mathrm{~S} 1$ platform with 50-bp paired-end reads.

Transcriptome quantification. Reads were assessed for quality with FastQC, trimmed and qualityfiltered with Trimmomatic (57), depleted of reads mapping to the human genome (GRCh38.p13) with STAR (58) and mapped and quantified with Salmon (59) using the Plasmodium falciparum 3D7 transcriptome (ASM276v2). Quantification files of parasite gene expression were summarized with the tximport package in $\mathrm{R}(60)$. Principal-component analysis data were generated in R with the plotPCA function from DESeq2 and then plotted with ggplot2. For heat map visualization, transcripts per million (TPM) were normalized around the mean for each transcript over the time series and depicted as a $z$ score of standard deviations from the mean.

Peak shift analysis. Analyses of the shift of transcript peak were conducted by first identifying for each transcript in each time series the time point at which the transcript achieved its maximum expression and then computing for each transcript the difference between time series of the transcript's peak. We repeated this approach using only transcripts with a single peak over the time series, which were identified using the find_peaks function from the signal processing submodule in SciPy. We compared the distributions of peak shift values between samples using Kruskal-Wallis one-way analysis of variance.

Differential expression analysis. All differential expression analyses were performed with the DESeq2 (61) R package using unnormalized read counts per transcript. Transcripts were considered differentially expressed if, as determined by DESeq2 with a false-discovery rate set to 0.05 , their adjusted $P$ value was $<0.05$ and $\left|\log _{2}(\mathrm{HbAS} / \mathrm{HbAA})\right|$ was $>\log _{2}(1.5)$ in the time series and, with a false-discovery rate set to 0.1 , the adjusted $P$ value was $<0.05$ and $\left|\log _{2}(\mathrm{HbAS} / \mathrm{HbAA})\right|$ was $>\log _{2}(1.5)$ in the field samples.

Gene ontology enrichment analysis. Gene ontology enrichment analysis was performed with GOATOOLS (17). Gene IDs and associated GO IDs were downloaded from NCBI (ftp://ftp.ncbi.nlm.nih .gov/gene/DATA/gene2go.gz), and $P$. falciparum's annotations were extracted with the taxonomic identifier 36329. Using the transcripts identified as differentially expressed by DESeq2 according to the conditions described above as input, gene ontology terms were accepted for Benjamini-Hochberg-adjusted $P$ values of $<0.05$.

Analyses of transcript temporal regulation. Dynamic time warping (DTW) of time series transcript expression data was computed with the tslearn toolkit (62). We considered transcripts with mean DTW scores across $\beta$-globin replicates that exceeded two standard deviations above the mean DTW scores of all transcripts in HbAA versus HbAS comparisons temporally dysregulated.

We used the temporal alignment Kendall-Tau algorithm (TAKT) (18) to identify transcripts within each parasite that had similar dynamics but were temporally shifted between $\beta$-globin types during the 
ring stage (18). Measures of similarity and associated significance scores were produced for a gene between similar $\beta$-globin type replicates and a $P$ value of $\leq 0.05$ was used to identify transcripts with similar dynamics across all $\beta$-globin types within each parasite.

Stage classification of in vivo parasites. We identified the set of transcripts shared between 3D7 and FUP from our in vitro time series that peaked within rings, trophozoites, or schizonts and then selected transcripts that were highly expressed at their peak, discarding those that did not exceed 1,000 TPM. We also estimated parasite stage with a previously published mixture model (20). Our count data were converted to reads per kilobase per million (RPKM) and $\log _{2}$ transformed to match the staged expression data (63) used for stage estimation.

Data availability. The RNA-seq data are available in GEO (accession no. GSE163144; BioProject no. PRJNA685106). All scripts, protocols, analyses, and supplemental output are available on GitHub (64). See Supplemental Text S1 for more experimental, processing, and analytic detail.

\section{SUPPLEMENTAL MATERIAL}

Supplemental material is available online only.

TEXT S1, DOCX file, 0.04 MB.

\section{ACKNOWLEDGMENTS}

This work was supported by the National Institute of Allergy and Infectious Disease (R21 Al125988 to S.M.T.). J.W.S. was supported by the National Center for Advancing Translational Sciences (UL1TR002553) and J.E.V.P. by the Alfred Benzon Foundation.

We thank Marilyn Telen and Nirmish Shah (both of Duke University) for their assistance with blood collection and Tom Wellems and Chanaki Amaratunga (both of NIAID) for assistance with clinical study data. P. falciparum strains 3D7 (MRA-102, contributed by Daniel J. Carucci) and FUP UGANDA-PALO ALTO (MRA-915, contributed by T. Sam-Yellowe) were obtained from BEI Resources, NIAID, NIH. We are indebted to the Malian children and their guardians who participated in the field study and to our donors of normal and sickle-trait blood.

\section{REFERENCES}

1. Malaria Genomic Epidemiology Network. 2014. Reappraisal of known malaria resistance loci in a large multicenter study. Nat Genet 46: 1197-1204. https://doi.org/10.1038/ng.3107.

2. Taylor SM, Parobek CM, Fairhurst RM. 2012. Haemoglobinopathies and the clinical epidemiology of malaria: a systematic review and meta-analysis. Lancet Infect Dis 12:457-468. https://doi.org/10.1016/S1473-3099(12)70055-5.

3. Pasvol G. 1980. The interaction between sickle haemoglobin and the malarial parasite Plasmodium falciparum. Trans R Soc Trop Med Hyg 74: 701-705. https://doi.org/10.1016/0035-9203(80)90182-0.

4. Friedman MJ. 1978. Erythrocytic mechanism of sickle cell resistance to malaria. Proc Natl Acad Sci U S A 75:1994-1997. https://doi.org/10.1073/ pnas.75.4.1994.

5. Pasvol G, Weatherall DJ, Wilson RJ. 1978. Cellular mechanism for the protective effect of haemoglobin S against P falciparum malaria. Nature 274: 701-703. https://doi.org/10.1038/274701a0.

6. Archer NM, Petersen N, Clark MA, Buckee CO, Childs LM, Duraisingh MT. 2018. Resistance to Plasmodium falciparum in sickle cell trait erythrocytes is driven by oxygen-dependent growth inhibition. Proc Natl Acad Sci U S A 115:7350-7355. https://doi.org/10.1073/pnas.1804388115.

7. LaMonte G, Philip N, Reardon J, Lacsina JR, Majoros W, Chapman L, Thornburg CD, Telen MJ, Ohler U, Nicchitta CV, Haystead T, Chi J-T. 2012. Translocation of sickle cell erythrocyte microRNAs into Plasmodium falciparum inhibits parasite translation and contributes to malaria resistance. Cell Host Microbe 12:187-199. https://doi.org/10.1016/j.chom.2012.06.007.

8. Cyrklaff M, Srismith S, Nyboer B, Burda K, Hoffmann A, Lasitschka F, Adjalley S, Bisseye C, Simpore J, Mueller A-K, Sanchez CP, Frischknecht F, Lanzer M. 2016. Oxidative insult can induce malaria-protective trait of sickle and fetal erythrocytes. Nat Commun 7:13401. https://doi.org/10 .1038/ncomms13401.

9. Waldecker $M$, Dasanna AK, Lansche $C$, Linke $M$, Srismith $S$, Cyrklaff $M$, Sanchez CP, Schwarz US, Lanzer M. 2017. Differential time-dependent volumetric and surface area changes and delayed induction of new permeation pathways in $\mathrm{P}$ falciparum-infected hemoglobinopathic erythrocytes. Cell Microbiol 19:e12650. https://doi.org/10.1111/cmi.12650.

10. Cholera R, Brittain NJ, Gillrie MR, Lopera-Mesa TM, Diakité SAS, Arie T, Krause MA, Guindo A, Tubman A, Fujioka H, Diallo DA, Doumbo OK, Ho M,
Wellems TE, Fairhurst RM. 2008. Impaired cytoadherence of Plasmodium falciparum-infected erythrocytes containing sickle hemoglobin. Proc Natl Acad Sci U S A 105:991-996. https://doi.org/10.1073/pnas.0711401105.

11. Kilian N, Srismith S, Dittmer M, Ouermi D, Bisseye C, Simpore J, Cyrklaff M, Sanchez CP, Lanzer M. 2015. Hemoglobin S and C affect protein export in Plasmodium falciparum-infected erythrocytes. Biol Open 4:400-410. https://doi.org/10.1242/bio.201410942.

12. Lansche C, Dasanna AK, Quadt K, Fröhlich B, Missirlis D, Tétard M, Gamain B, Buchholz B, Sanchez CP, Tanaka M, Schwarz US, Lanzer M. 2018. The sickle cell trait affects contact dynamics and endothelial cell activation in Plasmodium falciparum-infected erythrocytes. Commun Biol 1:211. https:// doi.org/10.1038/s42003-018-0223-3.

13. Fairhurst RM, Bess CD, Krause MA. 2012. Abnormal PfEMP1/knob display on Plasmodium falciparum-infected erythrocytes containing hemoglobin variants: fresh insights into malaria pathogenesis and protection. Microbes Infect 14:851-862. https://doi.org/10.1016/j.micinf.2012.05.006.

14. Serjeant GR. 2013. The natural history of sickle cell disease. Cold Spring Harb Perspect Med 3:a011783. https://doi.org/10.1101/cshperspect.a011783.

15. Preston MD, Campino S, Assefa SA, Echeverry DF, Ocholla H, AmambuaNgwa A, Stewart LB, Conway DJ, Borrmann S, Michon P, Zongo I, Ouédraogo J-B, Djimde AA, Doumbo OK, Nosten F, Pain A, Bousema T, Drakeley CJ, Fairhurst RM, Sutherland CJ, Roper C, Clark TG. 2014. A barcode of organellar genome polymorphisms identifies the geographic origin of Plasmodium falciparum strains. Nat Commun 5:4052. https://doi .org/10.1038/ncomms5052.

16. Geiman QM, Meagher MJ. 1967. Susceptibility of a New World monkey to Plasmodium falciparum from man. Nature 215:437-439. https://doi.org/ 10.1038/215437a0.

17. Klopfenstein DV, Zhang L, Pedersen BS, Ramírez F, Warwick Vesztrocy A, Naldi A, Mungall CJ, Yunes JM, Botvinnik O, Weigel M, Dampier W, Dessimoz C, Flick P, Tang H. 2018. GOATOOLS: a Python library for Gene Ontology analyses. Sci Rep 8:10872. https://doi.org/10.1038/s41598-018 -28948-z.

18. Kelliher CM, Foster MW, Motta FC, Deckard A, Soderblom EJ, Moseley MA, Haase SB. 2018. Layers of regulation of cell-cycle gene expression in the 
budding yeast Saccharomyces cerevisiae. Mol Biol Cell 29:2644-2655. https://doi.org/10.1091/mbc.E18-04-0255.

19. Miller LH, Baruch DI, Marsh K, Doumbo OK. 2002. The pathogenic basis of malaria. Nature 415:673-679. https://doi.org/10.1038/415673a.

20. Tonkin-Hill GQ, Trianty L, Noviyanti R, Nguyen HHT, Sebayang BF, Lampah DA, Marfurt J, Cobbold SA, Rambhatla JS, McConville MJ, Rogerson SJ, Brown GV, Day KP, Price RN, Anstey NM, Papenfuss AT, Duffy MF. 2018. The Plasmodium falciparum transcriptome in severe malaria reveals altered expression of genes involved in important processes including surface antigen-encoding var genes. PLoS Biol 16:e2004328. https://doi.org/10.1371/journal.pbio.2004328.

21. Kumari S, Roy S, Singh P, Singla-Pareek SL, Pareek A. 2013. Cyclophilins: proteins in search of function. Plant Signal Behav 8:e22734. https://doi .org/10.4161/psb.22734.

22. Gavigan CS, Kiely SP, Hirtzlin J, Bell A. 2003. Cyclosporin-binding proteins of Plasmodium falciparum. Int J Parasitol 33:987-996. https://doi.org/10 .1016/S0020-7519(03)00125-5.

23. Külzer S, Charnaud S, Dagan T, Riedel J, Mandal P, Pesce ER, Blatch GL, Crabb BS, Gilson PR, Przyborski JM. 2012. Plasmodium falciparumencoded exported hsp70/hsp40 chaperone/co-chaperone complexes within the host erythrocyte. Cell Microbiol 14:1784-1795. https://doi.org/ 10.1111/j.1462-5822.2012.01840.x.

24. Joy S, Thirunavukkarasu L, Agrawal P, Singh A, Sagar BKC, Manjithaya R, Surolia N. 2018. Basal and starvation-induced autophagy mediates parasite survival during intraerythrocytic stages of Plasmodium falciparum. Cell Death Discov 4:43. https://doi.org/10.1038/s41420-018-0107-9.

25. Takala-Harrison S, Clark TG, Jacob CG, Cummings MP, Miotto O, Dondorp AM, Fukuda MM, Nosten F, Noedl $H$, Imwong M, Bethell D, Se Y, Lon C, Tyner SD, Saunders DL, Socheat D, Ariey F, Phyo AP, Starzengruber $P$, Fuehrer H-P, Swoboda P, Stepniewska K, Flegg J, Arze C, Cerqueira GC, Silva JC, Ricklefs SM, Porcella SF, Stephens RM, Adams M, Kenefic L, Campino S, Auburn S, MacInnis B, Kwiatkowski DP, Su X-Z, White NJ, Ringwald P, Plowe CV. 2013. Genetic loci associated with delayed clearance of Plasmodium falciparum following artemisinin treatment in Southeast Asia. Proc Natl Acad Sci U S A 110:240-245. https://doi.org/10.1073/ pnas. 1211205110.

26. Lee HJ, Georgiadou A, Walther M, Nwakanma D, Stewart LB, Levin M, et al. 2018. Integrated pathogen load and dual transcriptome analysis of systemic host-pathogen interactions in severe malaria. Sci Transl Med 10: eaar3619. https://doi.org/10.1126/scitranslmed.aar3619.

27. Petersen JEV, Saelens JW, Freedman E, Turner L, Lavstsen T, Fairhurst RM, Diakité M, Taylor SM. 2021. Sickle-trait hemoglobin reduces adhesion to both CD36 and EPCR by Plasmodium falciparum-infected erythrocytes. PLoS Pathog 17:e1009659. https://doi.org/10.1371/journal.ppat.1009659.

28. Rosenthal PJ. 2004. Cysteine proteases of malaria parasites. Int J Parasitol 34:1489-1499. https://doi.org/10.1016/j.ijpara.2004.10.003.

29. Aoki S, Li J, Itagaki S, Okech BA, Egwang TG, Matsuoka H, Palacpac NMQ, Mitamura T, Horii T. 2002. Serine repeat antigen (SERA5) is predominantly expressed among the SERA multigene family of Plasmodium falciparum, and the acquired antibody titers correlate with serum inhibition of the parasite growth. J Biol Chem 277:47533-47540. https://doi.org/10.1074/ jbc.M207145200.

30. Miller SK, Good RT, Drew DR, Delorenzi M, Sanders PR, Hodder AN, Speed TP, Cowman AF, de Koning-Ward TF, Crabb BS. 2002. A subset of Plasmodium falciparum SERA genes are expressed and appear to play an important role in the erythrocytic cycle. J Biol Chem 277:47524-47532. https:// doi.org/10.1074/jbc.M206974200.

31. Thomas JA, Collins CR, Das S, Hackett F, Graindorge A, Bell D, Deu E, Blackman MJ. 2016. Development and application of a simple plaque assay for the human malaria parasite Plasmodium falciparum. PLoS One 11:e0157873. https://doi.org/10.1371/journal.pone.0157873.

32. Collins CR, Hackett F, Atid J, Tan MSY, Blackman MJ. 2017. The Plasmodium falciparum pseudoprotease SERA5 regulates the kinetics and efficiency of malaria parasite egress from host erythrocytes. PLoS Pathog 13: e1006453. https://doi.org/10.1371/journal.ppat.1006453.

33. McCoubrie JE, Miller SK, Sargeant T, Good RT, Hodder AN, Speed TP, de Koning-Ward TF, Crabb BS. 2007. Evidence for a common role for the serine-type Plasmodium falciparum serine repeat antigen proteases: implications for vaccine and drug design. Infect Immun 75:5565-5574. https:// doi.org/10.1128/IAI.00405-07.

34. Chaal BK, Gupta AP, Wastuwidyaningtyas BD, Luah YH, Bozdech Z. 2010. Histone deacetylases play a major role in the transcriptional regulation of the Plasmodium falciparum life cycle. PLoS Pathog 6:e1000737. https:// doi.org/10.1371/journal.ppat.1000737.
35. Coetzee N, Sidoli S, van Biljon R, Painter H, Llinás M, Garcia BA, Birkholtz LM. 2017. Quantitative chromatin proteomics reveals a dynamic histone post-translational modification landscape that defines asexual and sexual Plasmodium falciparum parasites. Sci Rep 7:607. https://doi.org/10.1038/ s41598-017-00687-7.

36. Ponts N, Harris EY, Prudhomme J, Wick I, Eckhardt-Ludka C, Hicks GR, Hardiman G, Lonardi S, Le Roch KG. 2010. Nucleosome landscape and control of transcription in the human malaria parasite. Genome Res 20: 228-238. https://doi.org/10.1101/gr.101063.109.

37. Aidoo M, Terlouw DJ, Kolczak MS, McElroy PD, ter Kuile FO, Kariuki $S$, Nahlen BL, Lal AA, Udhayakumar V. 2002. Protective effects of the sickle cell gene against malaria morbidity and mortality. Lancet 359:1311-1312. https://doi.org/10.1016/S0140-6736(02)08273-9.

38. Le Hesran JY, Personne I, Personne P, Fievet N, Dubois B, Beyemé M, Boudin C, Cot M, Deloron P. 1999. Longitudinal study of Plasmodium falciparum infection and immune responses in infants with or without the sickle cell trait. Int J Epidemiol 28:793-798. https://doi.org/10.1093/ije/28 .4 .793 .

39. Stirnadel HA, Stockle M, Felger I, Smith T, Tanner M, Beck HP. 1999. Malaria infection and morbidity in infants in relation to genetic polymorphisms in Tanzania. Trop Med Int Health 4:187-193. https://doi.org/10 .1046/j.1365-3156.1999.43381.x.

40. Cyrklaff M, Sanchez CP, Kilian N, Bisseye C, Simpore J, Frischknecht F, Lanzer M. 2011. Hemoglobins $S$ and $C$ interfere with actin remodeling in Plasmodium falciparum-infected erythrocytes. Science 334:1283-1286. https://doi.org/10.1126/science.1213775.

41. Kilian N, Dittmer M, Cyrklaff M, Ouermi D, Bisseye C, Simpore J, Frischknecht F, Sanchez CP, Lanzer M. 2013. Haemoglobin S and C affect the motion of Maurer's clefts in Plasmodium falciparum-infected erythrocytes. Cell Microbiol 15:1111-1126. https://doi.org/10.1111/cmi.12102.

42. Lanzer M, Wickert $H$, Krohne G, Vincensini L, Braun Breton C. 2006. Maurer's clefts: a novel multi-functional organelle in the cytoplasm of Plasmodium falciparum-infected erythrocytes. Int J Parasitol 36:23-36. https://doi.org/10 .1016/j.jpara.2005.10.001.

43. Spycher C, Rug M, Pachlatko E, Hanssen E, Ferguson D, Cowman AF, Tilley L, Beck H-P. 2008. The Maurer's cleft protein MAHRP1 is essential for trafficking of PfEMP1 to the surface of Plasmodium falciparum-infected erythrocytes. Mol Microbiol 68:1300-1314. https://doi.org/10.1111/j.1365 $-2958.2008 .06235 . x$.

44. Cooke BM, Buckingham DW, Glenister FK, Fernandez KM, Bannister LH, Marti M, Mohandas N, Coppel RL. 2006. A Maurer's cleft-associated protein is essential for expression of the major malaria virulence antigen on the surface of infected red blood cells. J Cell Biol 172:899-908. https://doi .org/10.1083/jcb.200509122.

45. Pachlatko E, Rusch S, Müller A, Hemphill A, Tilley L, Hanssen E, Beck H-P. 2010. MAHRP2, an exported protein of Plasmodium falciparum, is an essential component of Maurer's cleft tethers. Mol Microbiol 77:1136-1152. https://doi.org/10.1111/j.1365-2958.2010.07278.x.

46. Hanssen E, Sougrat R, Frankland S, Deed S, Klonis N, Lippincott-Schwartz J, Tilley L. 2008. Electron tomography of the Maurer's cleft organelles of Plasmodium falciparum-infected erythrocytes reveals novel structural features. Mol Microbiol 67:703-718. https://doi.org/10.1111/j.1365-2958 .2007.06063.x.

47. Hirtzlin J, Farber PM, Franklin RM, Bell A. 1995. Molecular and biochemical characterization of a Plasmodium falciparum cyclophilin containing a cleavable signal sequence. Eur J Biochem 232:765-772. https://doi.org/10 $.1111 /$ j.1432-1033.1995.tb20871.x.

48. Mok S, Ashley EA, Ferreira PE, Zhu L, Lin Z, Yeo T, Chotivanich K, Imwong M, Pukrittayakamee S, Dhorda M, Nguon C, Lim P, Amaratunga C, Suon S, Hien TT, Htut $Y$, Faiz MA, Onyamboko MA, Mayxay M, Newton PN, Tripura R, Woodrow CJ, Miotto O, Kwiatkowski DP, Nosten F, Day NPJ, Preiser PR, White NJ, Dondorp AM, Fairhurst RM, Bozdech Z. 2015. Drug resistance. Population transcriptomics of human malaria parasites reveals the mechanism of artemisinin resistance. Science 347:431-435. https://doi.org/10 $.1126 /$ science. 1260403 .

49. Bell A, Roberts HC, Chappell LH. 1996. The antiparasite effects of cyclosporin A: possible drug targets and clinical applications. Gen Pharmacol 27:963-971. https://doi.org/10.1016/0306-3623(95)02148-5.

50. Mideo N, Reece SE, Smith AL, Metcalf CJ. 2013. The Cinderella syndrome: why do malaria-infected cells burst at midnight? Trends Parasitol 29: 10-16. https://doi.org/10.1016/j.pt.2012.10.006.

51. Adjalley SH, Scanfeld D, Kozlowski E, Llinas M, Fidock DA. 2015. Genomewide transcriptome profiling reveals functional networks involving the 
Plasmodium falciparum drug resistance transporters PfCRT and PfMDR1. BMC Genomics 16:1090. https://doi.org/10.1186/s12864-015-2320-8.

52. Smith LM, Motta FC, Chopra G, Moch JK, Nerem RR, Cummins B, Roche KE, Kelliher CM, Leman AR, Harer J, Gedeon T, Waters NC, Haase SB. 2020. An intrinsic oscillator drives the blood stage cycle of the malaria parasite Plasmodium falciparum. Science 368:754-759. https://doi.org/10.1126/ science.aba4357.

53. Rijo-Ferreira F, Acosta-Rodriguez VA, Abel JH, Kornblum I, Bento I, Kilaru G, Klerman EB, Mota MM, Takahashi JS. 2020. The malaria parasite has an intrinsic clock. Science 368:746-753. https://doi.org/10.1126/science.aba2658.

54. Lopera-Mesa TM, Doumbia S, Konaté D, Anderson JM, Doumbouya M, Keita AS, Diakité SAS, Traoré K, Krause MA, Diouf A, Moretz SE, Tullo GS, Miura K, Gu W, Fay MP, Taylor SM, Long CA, Diakité M, Fairhurst RM. 2015. Effect of red blood cell variants on childhood malaria in Mali: a prospective cohort study. Lancet Haematol 2:E140-E149. https://doi.org/10.1016/ S2352-3026(15)00043-5.

55. Venkatesan M, Amaratunga C, Campino S, Auburn S, Koch O, Lim P, Uk S, Socheat D, Kwiatkowski DP, Fairhurst RM, Plowe CV. 2012. Using CF11 cellulose columns to inexpensively and effectively remove human DNA from Plasmodium falciparum-infected whole blood samples. Malar J 11:41. https://doi.org/10.1186/1475-2875-11-41.

56. Cranmer SL, Magowan C, Liang J, Coppel RL, Cooke BM. 1997. An alternative to serum for cultivation of Plasmodium falciparum in vitro. Trans R Soc Trop Med Hyg 91:363-365. https://doi.org/10.1016/s0035-9203(97)90110-3.
57. Bolger AM, Lohse M, Usadel B. 2014. Trimmomatic: a flexible trimmer for Illumina sequence data. Bioinformatics 30:2114-2120. https://doi.org/10 $.1093 /$ bioinformatics/btu170.

58. Dobin A, Davis CA, Schlesinger F, Drenkow J, Zaleski $C$, Jha $S$, Batut $P$, Chaisson M, Gingeras TR. 2013. STAR: ultrafast universal RNA-seq aligner. Bioinformatics 29:15-21. https://doi.org/10.1093/bioinformatics/bts635.

59. Patro R, Duggal G, Love MI, Irizarry RA, Kingsford C. 2017. Salmon provides fast and bias-aware quantification of transcript expression. Nat Methods 14:417-419. https://doi.org/10.1038/nmeth.4197.

60. Soneson C, Love MI, Robinson MD. 2015. Differential analyses for RNA seq: transcript-level estimates improve gene-level inferences. F1000Res 4 : 1521. https://doi.org/10.12688/f1000research.7563.1.

61. Love MI, Huber W, Anders S. 2014. Moderated estimation of fold change and dispersion for RNA-seq data with DESeq2. Genome Biol 15:550. https://doi.org/10.1186/s13059-014-0550-8.

62. Tavenard RFJ, Vandewiele G, Divo F, Androz G, Holtz C, Payne M, Yurchak R, Russwurm M, Kolar K, Woods E. 2017. tslearn: a machine learning toolkit dedicated to time-series data. J. Machine Learning Res 21:1-6.

63. López-Barragán MJ, Lemieux J, Quiñones M, Williamson KC, Molina-Cruz A, Cui K, Barillas-Mury C, Zhao K, Su X-z. 2011. Directional gene expression and antisense transcripts in sexual and asexual stages of Plasmodium falciparum. BMC Genomics 12:587. https://doi.org/10.1186/1471-2164-12-587.

64. Saelens JW. 2021. Sickle-trait-RNAseq. https://github.com/duke-malaria -collaboratory/Sickle-trait-RNAseq. 